\title{
Towards polymer-based organic thermoelectric generators
}

Olga Bubnova and Xavier Crispin

The self-archived postprint version of this journal article is available at Linköping University Institutional Repository (DiVA):

http:// urn.kb.se/ resolve?urn=urn:nbn:se:liu:diva-85293

N.B.: When citing this work, cite the original publication.

Bubnova, O., Crispin, X., (2012), Towards polymer-based organic thermoelectric generators, Energy \& Environmental Science, 5(11), 9345-9362. https:// doi.org/ 10.1039/ c2ee22777k

Original publication available at:

https:// doi.org/ 10.1039/ c2ee22777k

Copyright: Royal Society of Chemistry

http:// www.rsc.org/ 


\title{
Towards Polymer-based Organic Thermoelectric Generators
}

\author{
Olga Bubnova and Xavier Crispin \\ Department of Science and Technology, \\ Campus Norrköping, Linköping University, \\ S-60174 Norrköping, Sweden,
}

\begin{abstract}
In response to the thread of environmental and ecological degradation along with projected fossil fuel depletion the active search for efficient renewable energy conversion technologies has been attempted in various research areas including the field of thermoelectrics. Despite the availability of considerable amounts of waste and natural heat stored in warm fluids $\left(<250^{\circ} \mathrm{C}\right)$ a lack of environmentally friendly materials with high natural abundance, low manufacturing cost and high thermoelectric efficiency impedes the widespread use of thermoelectric generators for energy harvesting on a large scale. In this perspective, we examine the possibility of using organic conducting polymers in thermoelectric applications. We provide an overview of the background and the key concepts of organic thermoelectrics and illustrate some of the first prototypes of polymer-based organic thermoelectric generators.
\end{abstract}

\section{Broader context}

The sun is the largest readily available renewable energy source potentially capable of meeting the growing worldwide demand in electricity ( 13TW in 2012, 26TW in 2050). Its irradiating power at the surface of the earth is $\sim 10^{5} \mathrm{TW}, 600 \mathrm{TW}$ of which could be practically used. The maximum conversion efficiency of solar energy into electricity via solar thermal plants or solar cells is estimated at $40 \%$, whereas a large amount of energy in the form of natural heat remains unused. Thermoelectric generators (TEGs) can transform a small fraction of this heat into electricity. In years to come, depending on the population growth and climate conditions, the integration of solar cells and TEGs technologies shall be considered as one of the essential parts of more efficient global energy conversion. As for now, the electricity production largely relies on fossil fuels and nuclear energy, while the fraction of electricity produced by solar energy is negligible The transformation of these primary energy sources into electricity or mechanical work (transport, electricity production, process industries) accompanied by substantial heat loses $(\sim 60 \%)$. About $50 \%$ of the waste heat is stored in large volume warm fluids $\left(\mathrm{T}<250^{\circ} \mathrm{C}\right)$ and as yet no viable technology exists to produce electricity from this low energy density heat. Thermoelectric generators could offer a complimentary solution to Rankine cycles.. Today's thermoelectric materials of choice for low temperature energy conversion are bismuth antimony telluride alloys. The constituting atomic elements have a low natural abundance and therefore expensive. Additionally, low natural abundance goes hand in hand with some level of toxicity for the environment. Thereby, in today's scenario it is unthinkable to enable a widespread use of thermoelectric installations for waste heat recovery. In that context, widely available, although less efficient organic thermoelectric materials have become the subject of interest to scientific community. The synthesis of such materials can be readily 


\section{Introduction}

The thermoelectric phenomena involves the simultaneous presence of both electrical and thermal currents. Three distinct thermoelectric effects (Seebeck, Peltier and Thomson effects) are identified and are known to be interrelated through Kelvin relations. With the aid of the transport coefficients, thermoelectric behavior can be expressed by:

$$
\begin{aligned}
& \vec{i}=\sigma(\vec{E}-\alpha \vec{\nabla} T) \\
& \vec{q}=\alpha T \vec{i}-\lambda \vec{\nabla} T
\end{aligned}
$$

or equivalently:

$$
\left(\begin{array}{l}
\vec{i} \\
\vec{s}
\end{array}\right)=\left(\begin{array}{ll}
\sigma & \sigma \alpha \\
\sigma \alpha & \lambda / T
\end{array}\right)\left(\begin{array}{l}
\vec{E} \\
-\vec{\nabla} T
\end{array}\right)
$$

Here $E$ is the electric field, $i$ is the electric current density, $q$ is the heat current density, $s$ is the entropy current, $T$ is the temperature, $\sigma$ is the electrical conductivity, $\alpha$ is the Seebeck coefficient and $\lambda$ is the thermal conductivity at zero electric field condition. The off diagonal elements of the transport matrix are identical due to the Onsager reciprocal relations.

$$
\alpha=\left(\frac{d V}{d T}\right)_{I=0}
$$

The Seebeck coefficient is the open circuit voltage obtained between the two ends of a material submitted to a temperature gradient. The Seebeck coefficient of a material A ( $\alpha$ A) cannot be measured without the creation of two junctions submitted to a temperature gradient $\Delta \mathrm{T}$ with another material $\mathrm{B}$ of well-known Seebeck coefficient $\alpha$ B. The measure thermovoltage produced between the two junctions $\mathrm{V}=\Delta \mathrm{T}\left(\alpha_{\mathrm{B}}-\alpha_{\mathrm{A}}\right)$, allows determining $\alpha$ A. Calibration can be realized with a superconductor B since $\alpha_{\mathrm{B}}=0 \mathrm{~V} / \mathrm{K}$. 


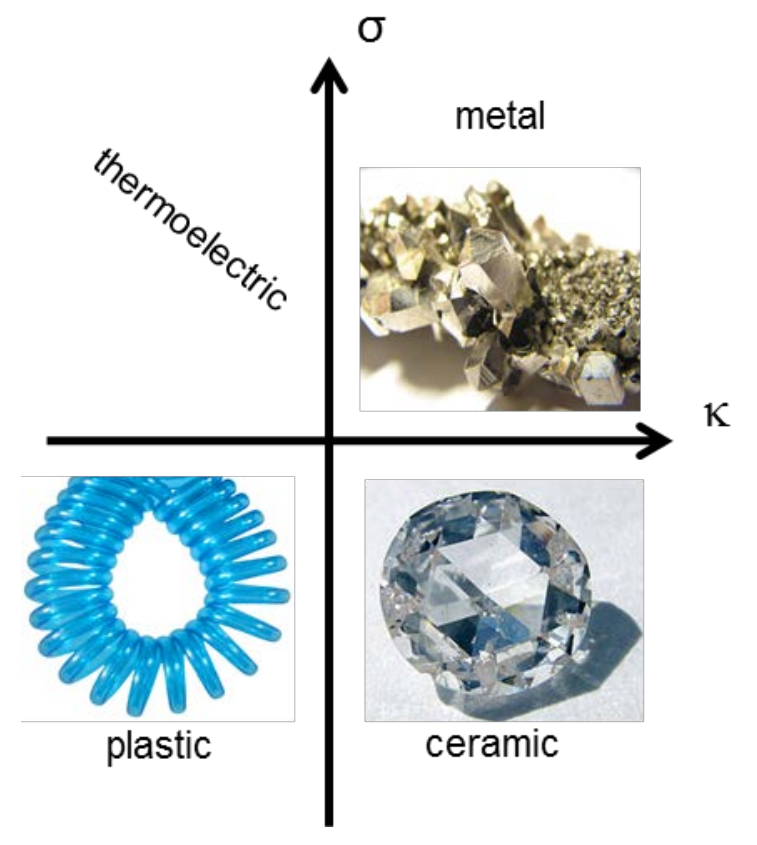

Fig.1: Different materials classified with respect to their thermal $\kappa$ and electrical $\sigma$ conductivities.

As for now, the thermoelectric energy conversion technologies have been targeting a number of rather exotic market niches and haven't made any serious impact on the energy and environmental issues. Peltier coolers, for example, are used in portable fridges or in photodetectors to reduce the noise ${ }^{1}$. Micro-Peltier coolers are attracting a lot of attention as a solution to discard the excess heat produced by Si-chip in computers ${ }^{2}$. Thermoelectric generators have been in development since the early sixties as a durable power source for satellites and spacecrafts. In these devices the heat produced upon radioisotopic decay ${ }^{3}$ is used for energy transformation. The body's heat is known to be used as a source of thermal energy for small micro-thermoelectric generators to power a wristwatch or some other low power electronic devices ${ }^{4}$. The concept of thermo-induced voltage $\mathrm{V}=\alpha \Delta \mathrm{T}$, better known as Seebeck voltage underlies the operation principle of thermocouple thermometers and temperature sensors.

A thermoelectric generator (TEG) is a device that produces an electrical current I or electrical power $\mathrm{P}=\mathrm{VI}$ from a temperature gradient $\Delta \mathrm{T}$. The thermoelectric material must be electro-conductive and produce a significant Seebeck voltage in order to ensure efficient thermoelectric power generation. Seebeck coefficients can be as small as few $\mu \mathrm{V} / \mathrm{K}$ for metals and as large as several $\mathrm{mV} / \mathrm{K}$ for electrical insulators ${ }^{5}$, hence thermoelectric materials are typically semiconductors or semi-metals with a combination of reasonable electrical conductivity and Seebeck coefficient. A TEG is composed of many thermocouples connected electrically in series and thermally in parallel in order to achieve a thermo-induced potential of few volts to power small electronic devices, motors, or to charge a battery (Fig. 2a). The device is sandwiched between ceramic layers to avoid short-circuiting between the metal interconnects and to ensure a good thermal exchange with the surroundings. The additive effect of the thermo-voltage created by 
each thermocouple is only possible if their legs are connected as depicted in Fig. 2. The ntype leg has a negative Seebeck coefficient, i.e. the electrons thermally diffuse to the cold side, while in the p-type leg a temperature gradient causes the propagation of holes towards the lower energy region.. An electromotive force is induced in a circuit as a force acting against further charge carrier migration.
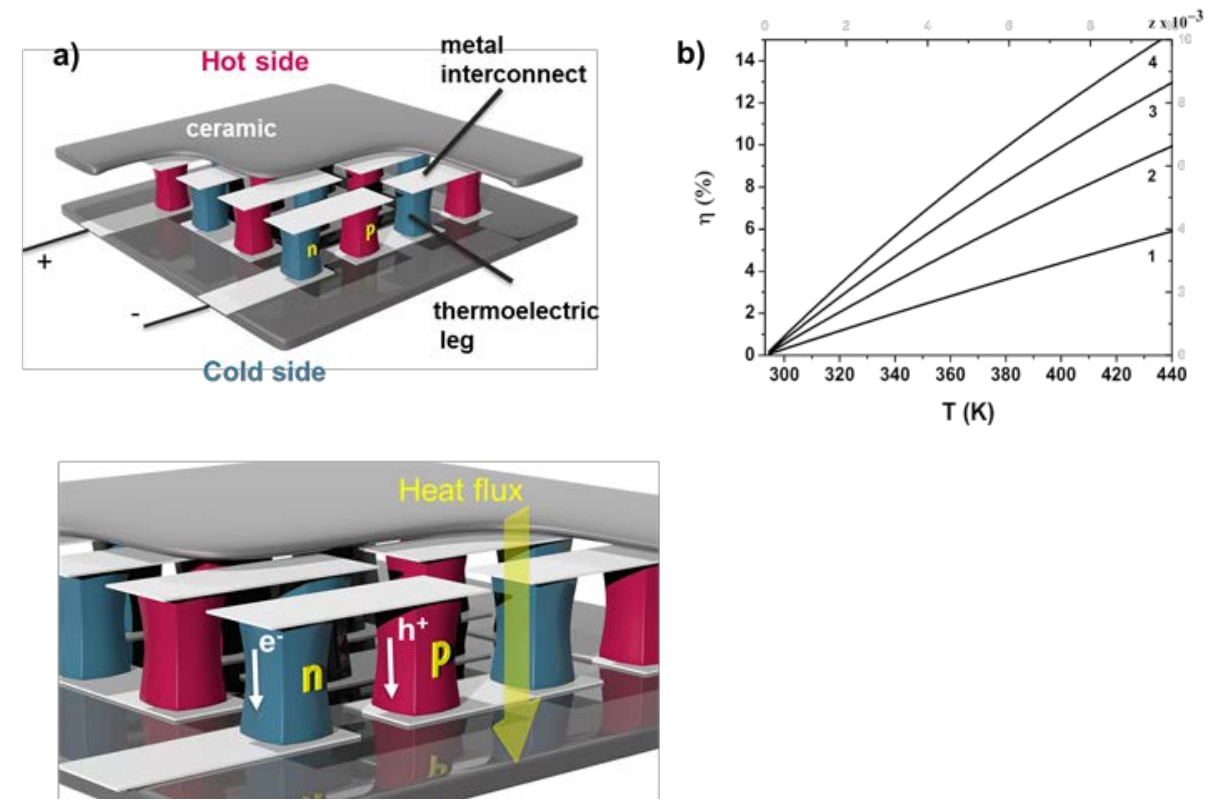

Fig. 2: Sketch of a thermoelectric module composed of p-type and n-type legs (left). The conversion efficiency of the module is plotted on the right hand side as function of the temperature for various $\mathrm{Z}$ values. $\mathrm{T}$ is the temperature of the hot side, while the cold side is maintained at $293 \mathrm{~K}$.

In order to attain significant Seebeck voltage V large temperature gradient needs to be established across the thermocouple. This condition can only be fulfilled on account of low thermal conductivity of a thermoelectric material. Thermal insulators are typically amorphous or semi-crystalline, such as glasses $\left(\kappa=\sim 1.1-1.5 \mathrm{WK}^{-1} \mathrm{~m}^{-1}\right)$ or plastics $(\kappa=\sim 0.1$ $\left.0.7 \mathrm{WK}^{-1} \mathrm{~m}^{-1}\right)^{6}$. The latter is not appropriate for thermoelectrics because of the low electrical conductivity. On the other hand, materials characterized by high electrical conductivity $(\sigma>1000 \mathrm{~S} / \mathrm{cm})$, such as metals or highly doped semiconductors are not ideal either due to their high thermal conductivities and low Seebeck coefficients. Hence, thermoelectric materials constitute a special class of materials with low thermal conductivity and high electrical conductivity (Fig. 1); often described as "a phonon-glass and electron-crystal" material ${ }^{7}$. Three key material properties are combined in the socalled dimensionless thermoelectric figure-of-merit, $\mathrm{ZT}=\left(\sigma \alpha^{2} / \kappa\right) \mathrm{T}$. The importance of this physical quantity lies in its direct relationship with the efficiency of a thermoelectric material or device. ZT quantifies the ratio between the thermal energy passing through the thermoelectric element and the electrical energy produced. The maximum theoretical 
efficiency is proportional to $(\mathrm{ZT}+1)^{1 / 2}$ and plotted in Fig. $2 \mathrm{~b}$ as a function of temperature $\mathrm{T}$ for various values of $\mathrm{Z}\left(\mathrm{T}_{\mathrm{C}}=293 \mathrm{~K}\right)$. A thermoelectric generator consisting of two legs with $\mathrm{ZT}=1$ is expected to reach $5 \%$ efficiency if the temperature difference between its hot and cold sides is set to $100 \mathrm{~K}$. The best thermoelectric materials possess ZT values around unity. One of the key strategies to optimize ZT in inorganic conductors is to reduce their thermal conductivity via introduction of nanostructures acting as phononscattering centers. Bismuth chalcogenides are found to be the best materials for room temperature applications with $\mathrm{ZT}>1^{8}$. For higher temperatures, inorganic clathrates (e.g. $\mathrm{ZT}=0.87$ at $\mathrm{T}=870 \mathrm{~K}$ ) ${ }^{9}$ and Half Heusler alloys (e.g. $\mathrm{ZT}=0.6$ at $\mathrm{T}=800 \mathrm{~K}{ }^{10}$ ) have shown assuring thermoelectric efficiency. The study of skutterudite materials (e.g. ZT=1.4 at T= $900 \mathrm{~K}^{10,{ }^{11}}$ ) that can potentially be extremely efficient is on the way. Promising thermoelectric materials based on atoms of high natural abundance and low toxicity, such as silicides (e.g. $\mathrm{ZT}=0.62$ at $800 \mathrm{~K}$ for p-type manganese silicides) ${ }^{12}$ and oxides (e.g. ZT= 0.87 at $973 \mathrm{~K}$ for $\left.\left(\mathrm{Ca}_{2} \mathrm{CoO}_{3}\right)_{0.7} \mathrm{CoO}_{2}\right)^{13}$, are currently under scientific scrutiny as they are believed to be suitable for the production of TEGs in vehicles, incineration plants, etc ${ }^{14}$. However no efficient thermoelectric materials of high abundance have been found for low temperature applications $\left(<500^{\circ} \mathrm{K}\right)$. In order to recover energy from low energy density sources (whether it's waste or natural heat) thermoelectric installations should spread over vast areas.. A substantial reduction in material and manufacturing costs is needed to enable a wide implementation of thermoelectric generators for energy harvesting (cogeneration).

In this review, we investigate the potential of a new class of thermoelectric materials: electrically conducting polymers characterized by low thermal conductivity and decent electrical conductivity. Besides, there some other arguments in their favor such as wide availability of polymers and polymerization techniques, their low toxicity and inexpensive processibility at low temperature. In the first part of the review, we explain the basic properties of conducting polymers: their chemical and electronic structures, as well as their electrical, thermal and thermoelectric properties. In the second part, we go through recent developments in "organic thermoelectrics" and demonstrate early prototypes of organic thermoelectric generators. Finally, we propose to explore some of the available strategies for further material's efficiency improvement and device fabrication processes.

\section{Conducting polymers}

\subsection{Undoped conjugated polymers}

Unlike saturated insulating polymers with $\mathrm{sp}^{3}$ hybridized carbons, in conjugated polymers such as trans-polyacetylene, the carbon atoms are $\mathrm{sp}^{2}$ hybridized. It means that three out of four electrons in the outer shell of each carbon atom form three $\sigma$-bonds that form the planar skeleton of the polymer chain. Each carbon atom contributes with one $2 p$ electron, which consequently becomes delocalized in $\pi$-orbitals with an electronic density 
dominant in the plane perpendicular to the $\sigma$-skeleton. The stabilization due to the formation of those delocalized $\pi$-orbitals, i.e. $\pi$-bond, results in large torsion barriers. Therefore, conjugated chains are more rigid than saturated chains ${ }^{15}$. Due to the onedimensional periodic structure, the $\pi$-orbitals can be described as Bloch functions characterized with a k-vector related to the wave-length of the delocalized $\pi$-electron. Fig. 3 displays the energy dispersion of the filled valence $\pi$-band and the empty conduction $\pi^{*}$-band for trans-polyacetylene. The presence of an alternation between single and double bonds emerges as a result of so-called Peierls instability ${ }^{16}$ which underlies the band gap formation. Saturated polymers are transparent with a large band gap between $\sigma-$ bands; in contrast, conjugated polymers have a band gap between $1 \mathrm{eV}$ and $4 \mathrm{eV}^{17}$ and appear as colored semiconducting plastics. It is difficult to inject a charge carrier (extra electron or deficient electron, i.e. hole) into a saturated polymer owing to its large band gap. Moreover it strongly destabilizes the polymer's structure (bond cleavage) preventing the injected charge from being transported. In a conjugated polymer, charge carriers are easily injected and efficiently transported through the $\pi$-orbitals; while the chain structure is maintained by the $\sigma$-skeleton.

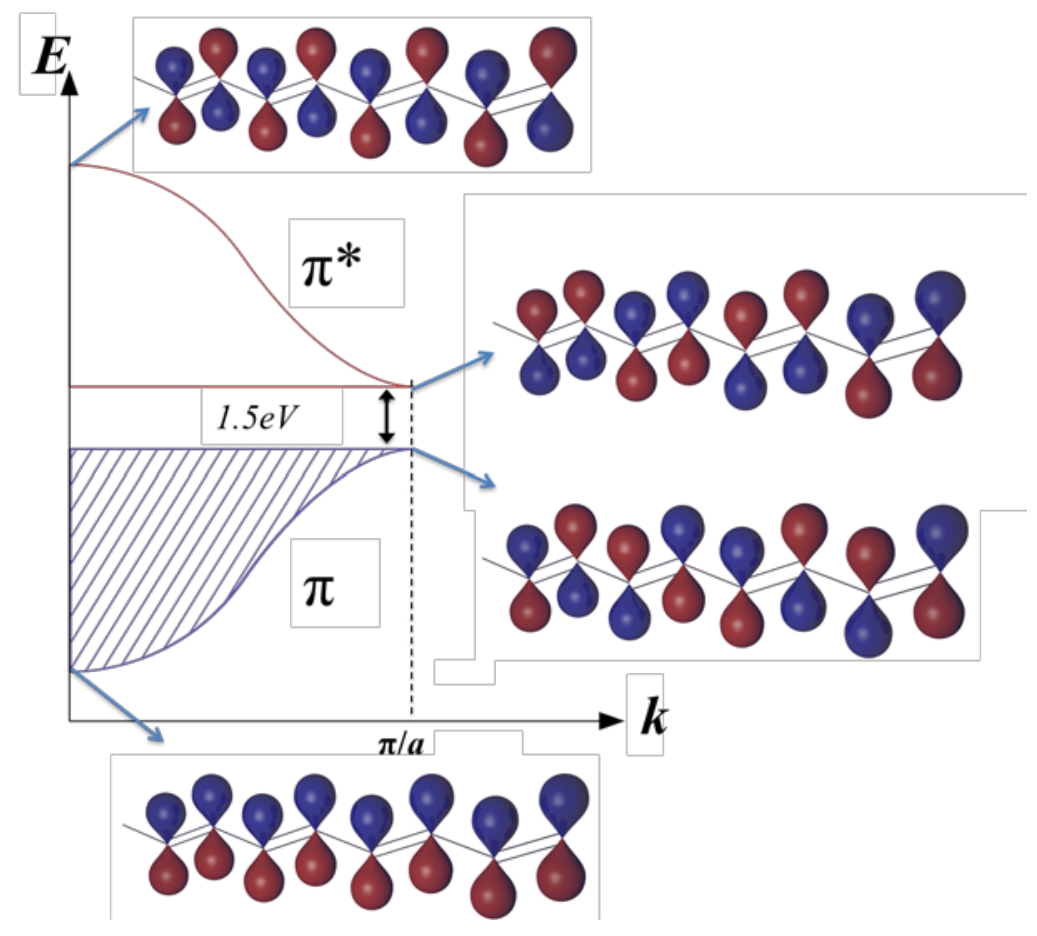

Fig. 3: Energy dispersion of the valence and conduction $\pi$-bands for trans-polyacetylene. The bonding scheme of the molecular orbitals is seen as combination of atomic $2 \mathrm{p}_{\mathrm{z}}$ orbitals pointing perpendicular to the skeleton of the polymer chain. 
<smiles>CC1(C)C=CC(C)(C)C1</smiles>

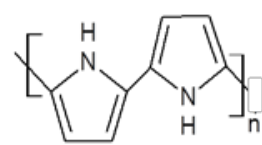<smiles>OCCOc1c(-c2sc(C(F)(F)F)c3c2OCCO3)sc2c1OCCO2</smiles>

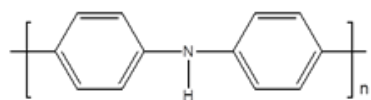

trans-polyacetylene

polypyrrole

poly(3,4-ethylenedoxythiophene)

\section{polyaniline}

Fig. 4: Chemical structure of conjugated polymers commonly used in their doped form as synthetic metals.

The molecular structures of some conjugated polymers frequently used as conducting polymers are depicted in Fig. 4, among them poly(acetylene), poly(aniline), poly(pyrrole) and the poly(thiophenes). A conjugated polymer chain typically comprises a few hundreds of repeating units (monomers), which is significantly shorter than the length of a non-conjugated polymer chain. Besides, they are nearly insoluble ${ }^{14}$ unless flexible side-chain substituents are introduced. Significant synthesis efforts have been devoted to solubilize semiconducting polymers in order to form homogeneous thin films from solution, which is not easy to achieve with small conjugated molecules provided their tendency to arrange in crystals ${ }^{15}$. From a technological point of view, the solubility of conjugated polymer enables electronics manufacturing with printing technologies (printed electronics) ${ }^{18}$. The rigidity of the conjugated chains and their ability to form weak covalent interactions, called $\pi-\pi$ interactions ${ }^{19} 20$ favor the chains stacking, the aggregates creation in the solution, and the formation of crystalline domains in polymer films. The molecular structure of the monomer unit can be modified to either promote stacking for high charge carrier mobility in transistors ${ }^{21}$, or prevent stacking to increase luminescence efficiency in light emitting diodes ${ }^{22}$. Hence, the charge carrier transport in conjugated polymer devices is not one-dimensional, but rather two or even threedimensional. In disordered organic semiconductors, the electronic coupling between adjacent chains is often limiting the transport at the macroscopic level. The average electronic coupling, or transfer integral $t$, is lower than in a single organic crystal $(\mathrm{t}<0.05 \mathrm{eV})^{22,23}$. To a first approximation, it provides the width $\mathrm{W}=4 \mathrm{t}$ of the inter-chain band created by $\pi-\pi$ interactions ${ }^{24} 25$. The residence time of the charge carrier is estimated at $\tau=\hbar / \mathrm{W}^{26}$. If $\tau$ is larger than the typical time for a vibrational mode $\left(10^{-13} \mathrm{~s}\right)$, which is the case when $\mathrm{W}>0.2 \mathrm{eV}$, the polymer chain carrying the charge carrier has time to relax its structure locally and adapt to the new electronic density altered by the presence of the charge carrier. The charge carrier is then self-trapped in the form of a local defect, a polaron. The electrical transport takes place via hopping of polarons between localized 
states. The mobility of the charge carrier depends on the polaron binding energy, the energy disorder and the positional disorder. If $\tau$ is smaller than typical vibrational time, the charge carrier is delocalized. The transport can be described as in inorganic crystalline semiconductors with the motion of a charge in a band with an effective mass. Such a phenomenon is observed in organic single crystals at low temperature since upon cooling, the transfer integral increases and the charge carriers become delocalized at a certain temperature ${ }^{26}$. The mobility in conjugated polymers varies from $10^{-5} \mathrm{~cm}^{2} \mathrm{~V}^{-1} \mathrm{~s}^{-1}$ to $\sim 1$ $\mathrm{cm}^{2} \mathrm{~V}^{-1} \mathrm{~s}^{-1}$ for holes ${ }^{27}$ and electrons ${ }^{28}$. Interestingly, solution-processed conjugated polymers can self-organize so well that their mobility values become comparable to those of organic single crystals ( $\sim 10 \mathrm{~cm}^{2} \mathrm{~V}^{-1} \mathrm{~s}^{-1}$ for holes ${ }^{29}$ and $\sim 1 \mathrm{~cm}^{2} \mathrm{~V}^{-1} \mathrm{~s}^{-1}$ for electrons $\left.{ }^{30}\right)$.

\subsection{Doped conjugated polymers}

Conducting polymers were discovered in the late seventies when it was demonstrated for the first time that poly(acetylene) exposed to iodine vapors could become conductive through a redox reaction ${ }^{31} 32,33$. As a result the electrical conductivity increased from $10^{-9} \mathrm{~S} / \mathrm{cm}$ to $10^{5} \mathrm{~S} / \mathrm{cm}$ followed by a formation of a metal-like low-weight and flexible material, which subsequently became known as "synthetic metal" ${ }^{33}$. Since then many conjugated polymers characterized by a tunable electrical conductivity have been synthesized. This phenomenon is sometimes called "doping” by analogy with inorganic semiconductors, although the major difference lies in the doping level (dopant concentrations in ppm for inorganics (around 1\%) and up to 35\% for conducting polymers). Hence, neutral or undoped conjugated polymers possess semiconducting properties, while oxidized or doped conjugated polymers are called conducting polymers because they show similarities with doped semiconductors or metals. Note that a reduction of a conjugated polymer is also possible, equivalent to a n-doping in inorganic materials, but in this form, the polymers are typically unstable ${ }^{34}$.

There are two possible ways to increase the doping level of a conjugated polymer to make it conducting. In case of electrochemical doping, the conjugated polymer is in contact with a metal electrode and an electrolyte. The extra charge carriers are provided by the metal electrode. Small counterions, i.e. ions with opposite charge, can penetrate in the polymer to maintain its electro-neutrality. This mass transfer is allowed due to weak van der Waals interactions within the polymer. The oxidation [reduction] of a conjugated molecule takes place at a specific applied electric potential, i.e. when the electrochemical potential of the metal electrons corresponds to the ionization potential [electron affinity] of the conjugated segment in the electrolyte medium ${ }^{35}$. The alternative method is the chemical doping or redox reaction. In that case, the polymer is exposed to a gas or dipped in a solution containing an oxidizing agent (molecule of high electron affinity), also called "dopant", that oxidizes the conjugated polymer. The reduced dopant transforms into a negative counterion, anion, neutralizing the positive charge introduced in the $\pi$-electron system of the polymer. Examples of such dopants are iodine $\mathrm{I}_{2}$ transforming in $\mathrm{I}_{3}{ }^{36}$, or a solution of the salt $\mathrm{NO}^{+} \mathrm{PF}_{6}{ }^{-37}$ where the $\mathrm{NO}$ gas is released while the $\mathrm{PF}_{6}{ }^{-}$anion 
becomes a counterion in the oxidized polymer. One form of polyaniline, the emeraldine base, is oxidized into the conducting emeraldine salt simply by acid treatment ${ }^{38}$. In this specific case of doping, the protons bound covalently to the nitrogen of polyaniline and its positive charge is transferred to the $\pi$-electron system. Finally, conducting polymers can directly be synthesized in their oxidized form by electropolymerization ${ }^{39}$ or chemical polymerization ${ }^{40}$. In that case, decreasing the oxidation level (undoping) starting from a highly oxidized polymer can be realized through an electrochemical or chemical reaction, using a reducing agent ${ }^{41}$.

The electrostatic interactions between the dopants and the counterions augment the cohesive energy of conducting polymers as compared to their undoped counterparts. Hence highly doped conducting polymers are typically insoluble. The common strategy for solubility enhancement lays in a dispersion formation via micelle-like particles either with a soluble polymeric counterion (polystyrene sulfonate for poly(3,4ethylenedioxythiophene), PEDOT-PSS) ${ }^{40}$ or using ionic surfactant serving as a counterion (camphorsulfonic acid for polyaniline, Pani-CSA) ${ }^{42}$. The presence of specific high boiling point solvents favors a better polymer chains organization and can increase the conductivity by several orders of magnitude ${ }^{43} 44$. This morphology effect is called "secondary" doping to distinguish it from the "primary" effect related to the oxidation (doping) level .
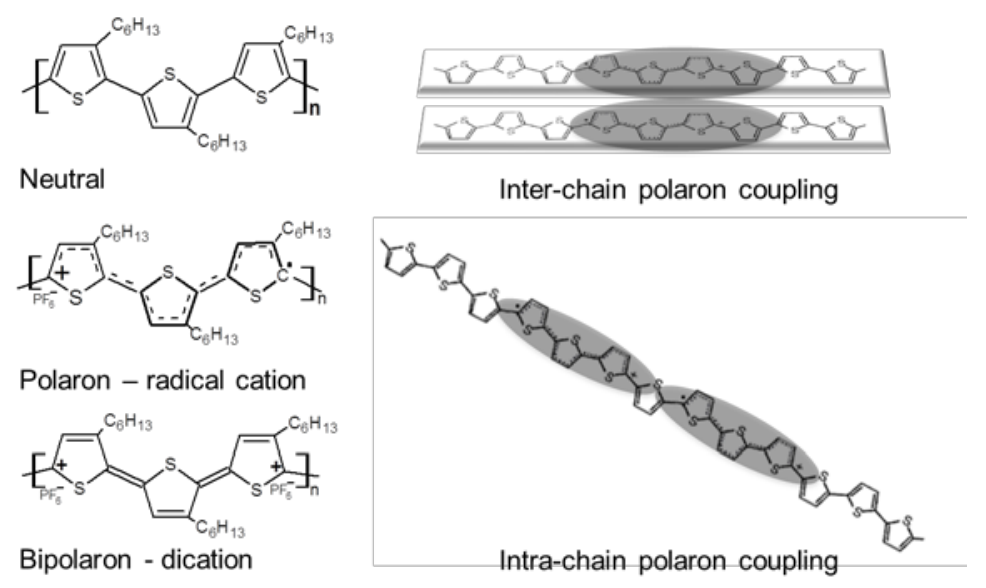

Fig. 5: Chemical structure of a neutral polythiophene chain, as well as a chain that carries a polaron and a bipolaron (left). At high doping level, the coupling between (bi)polarons can be either intra-chain or inter-chain and is at the origin of the creation (bi)polaronic bands (right). 


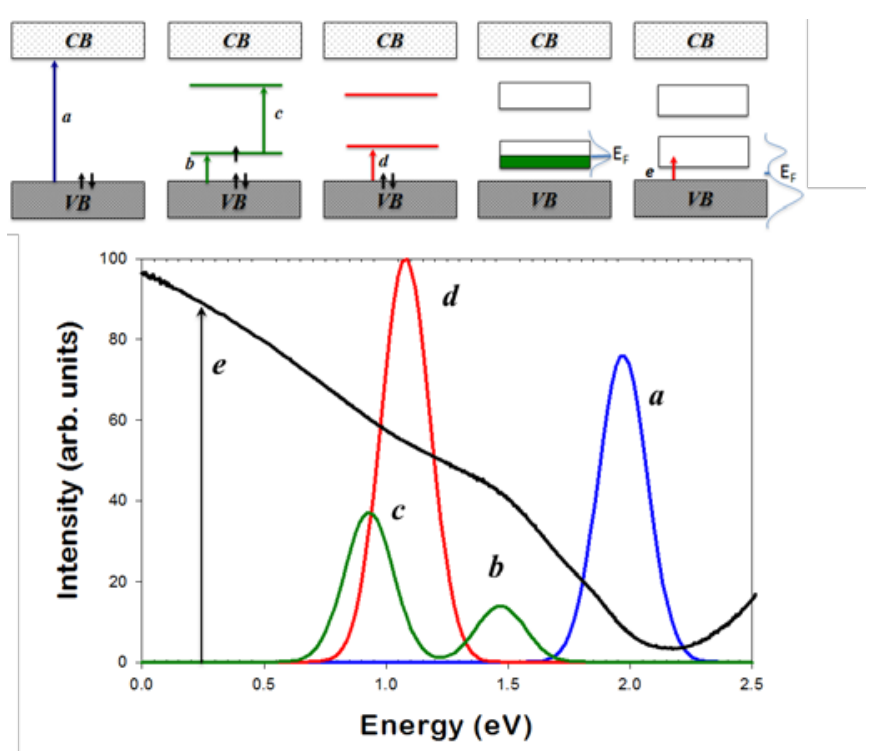

Fig. 6: Electronic structure for polymer chains at various oxidation state from left to right: a neutral chain, a chain carrying a polaron, a chain carrying a bipolaron, a chain or assembly of chains with a high concentration of polarons (intra chain or interchain polaron network), a chain or assembly of chains with a high concentration of bipolarons (intrachain or interchain bipolaron bands). The optical spectrum of these species is illustrated on the bottom.

The doping charges can be found in three possible distinct forms namely charged solitons, polarons and bipolarons depending on the chemical structure of the polymer and its doping level. Solitons are only found in trans-polyacetylene; as a manifestation of its high symmetry with two possible degenerate structures (a change in the bond length alternation does not change the energy of the polymer chain). This special case is not considered in this review. An example of polaron and bipolaron formation in polythiophene is depicted in figure 5. When an electron is removed from the top of the valence band, an antibonding character appears in some $\pi$-bonds and a distortion is induced locally in the structure of the chain. The quasi-particle composed of a positive charge associated with the lattice distortion is called a positive polaron by the physicists or delocalized radical cation by the chemists. The existence of those charge carriers can be identified by electron spin/paramagnetic resonance spectroscopy (ESR or EPR) since polarons carry a half-integer spin ${ }^{45}$. While the structure of the neutral chain is typically characterized by an aromatic character on cyclic monomers, the polaron distortion appears to have more of a quinoid character, which tends to permute the bond length alternation (double bonds become single bonds and vice versa). The unpaired electron lays on a half-filled electronic level above the valence band edge. The related antibonding level is an empty electronic level below the bottom of the conduction band (Fig. 6) ${ }^{46}$. This distortion extends over three to four monomer units depending on the proximity of the counterion. Positive bipolarons, or delocalized dications, are quasi-particles carrying two positive charges and associated with the same lattice defect. Bipolarons are formed rather than two polarons due to subtle energetics involving the Coulomb repulsion between the two charges, the cost in lattice distortion and the electrostatic stabilization 
due to counterions. The lattice distortion in a bipolaron is more pronounced ${ }^{34}$ than in a polaron with a clear quinoid structure resulting in two empty bipolaronic levels shifted further into the band gap (Fig. 6). The bipolaron [polaron] level is $0.75 \mathrm{eV}[0.5 \mathrm{eV}$ less stable than the top of the valence band and reflects a strong binding energy due to a significant electron-phonon coupling ${ }^{46}$. Bipolarons are not expected to be very mobile and participate in the transport by splitting into polarons under the influence of an electric field ${ }^{47}$. At high doping level, the wave functions of adjacent (bi)polarons, located either on the same chain or on the neighboring chains, start to overlap and lead to the formation of intra-chain ${ }^{48}$ or inter-chain ${ }^{49}$ (bi)polaron bands (Figs. 5, 6). The width of the bands increases with the doping level so much that the conducting polymers have a vanishingly small gap and may exhibit metallic-like electrical properties at room temperature ${ }^{42}$.

The evolution of the optical properties of a conjugated polymer subject to doping is illustrated in Fig. 6. This phenomenon is called electrochromism and is induced by electrochemical doping ${ }^{50}$. A wide band gap transparent conjugated polymer can become colored upon doping; likewise a low band gap conjugated polymer in its colored state becomes transparent when oxidized. A neutral polymer chain of planar symmetry displays a first optical transition from the top of the valence band to the bottom of the conduction band (transition a). Note that the terminology of "band" is often abandoned when describing optical properties because of the excitonic state created by an electron-hole pair, which is localized on a small segment of the polymer chain due to its strong binding energy (0.2-0.3 eV) and low dielectric constant ${ }^{51}$. Hence, the optical transition for a polymer chain is similar to that of an oligomer molecule of equivalent conjugation length. The low energy absorption feature is associated to a transition between the Highest Occupied Molecular Orbital and the Lowest Unoccupied Molecular Orbital (HOMOLUMO) transition (a). In a polymer film, the absorption features are broad, shifted and of different intensities compared to isolated chains in solution due to the aggregation between chains ${ }^{52}$. The optical transition dipoles of adjacent chains interact with one another and new transitions with inter-chain excitons occur ${ }^{22}$. Upon doping, new polaronic or bipolaronic states appear in the band gap of the polymer. Due to the planar symmetry of a polymer chain, these intra band gap states manifest themselves in new optical transitions (transitions b and c in case of a radical-cation (polaron) and a single transition d in case of a dication (bipolaron) on an isolated polymer chain (in solution)). In the solid state, the aggregation of chains relaxes the symmetry selection rules and more transitions are possible ${ }^{49}$. With additional shift and broadening effects, it becomes difficult to strictly identify transitions for a polaron and a bipolaron. The situation gets complex at high doping levels but (bi)polaron band formation shows typically a broad IR absorption (transition e) eventually resembling the Drude absorption of metals extending in the far IR ${ }^{42}$.

\subsection{Electrical conductivity}

In undoped conjugated polymers, the charge transport is reasonably described by phonon-assisted hopping between energy levels in a Gaussian disorder. When not 
bounded to a counterion, a polaron can hop to a next neutral segment under the influence of the electric field. The intrinsically very low charge carrier density in conjugated polymers $\left(<10^{20}|\mathrm{e}| \mathrm{m}^{-3}\right)^{53}$ is accountable for their low electrical conductivity $(\sigma=\mathrm{n}|\mathrm{e}| \mu$, where $\mathrm{n}$ is the charge carrier density, $|\mathrm{e}|$ is the elementary charge, $\mu$ is the mobility). To increase the electrical conductivity, doping is required. In the process of doping extra charge carriers are introduced into the polymer. Once on the chain, they get localized by the traps created by attractive Coulomb potentials of the counterions. Since the dielectric constant of organic materials is very low $(\varepsilon=3)^{54}$, the Coulomb traps are rather large in size reaching $20 \mathrm{~nm}$ in radius. A single isolated Coulomb trap includes several dozens of hopping sites. Hence, at low doping concentration, the mobility is suppressed by trapping. At higher counterion concentration/doping level (starting from $10^{17} \mathrm{~cm}^{-3}$ ) the Coulomb traps begin to overlap and the energy barriers between them tend to decrease (Fig. 7). It translates into energy disorder reduction of the $\pi$-orbitals involved in the charge transport and a significant charge mobility increase is expected (e.g. from $10^{-5} \mathrm{~cm}^{2} \mathrm{~V}^{-1} \mathrm{~s}^{-1}$ to $10^{-1}$ $\left.\mathrm{cm}^{2} \mathrm{~V}^{-1} \mathrm{~s}^{-1}\right)^{55}$.

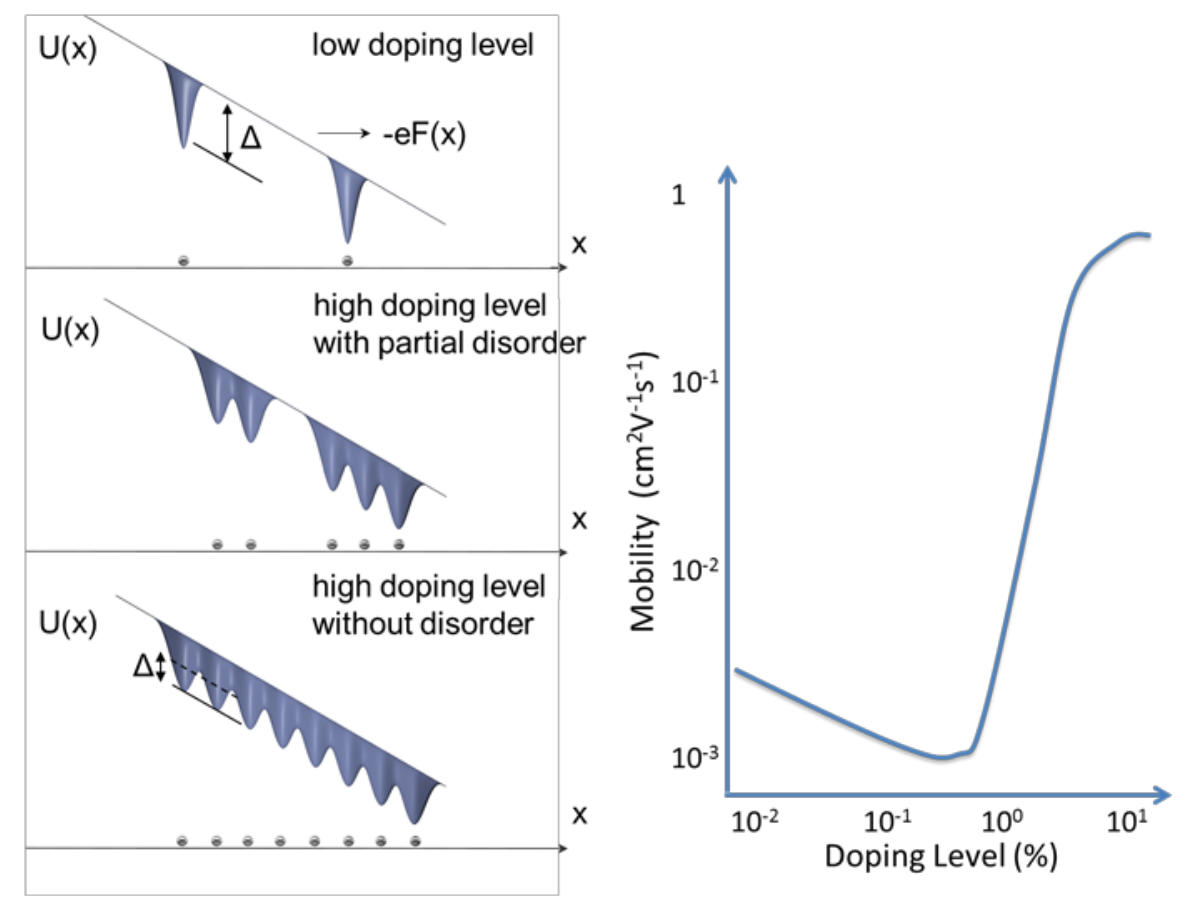

Fig.7: Electrostatic potential landscape $\mathrm{U}(\mathrm{x})$ and reduction of the energy barrier $\Delta$ for the charge transport under the influence of an external electric field $\mathrm{F}(\mathrm{x})$ created by the counterions for different doping levels and spatial disorder (left) and the effect of doping level on the mobility of charge carriers (right).

At high oxidation levels (up to 30\%) when the distance between counterions is reduced $(\approx 1.5 \mathrm{~nm})$ the electrostatic landscape becomes smooth, i.e. characterized by low energy barriers $(\Delta)$ facilitating charge hopping. The electrical conductivity reaches values up to several thousands of $\mathrm{S} / \mathrm{cm}^{56}$. The positional disorder along with the energetic 
disorder strongly affects the transport of charge carriers. There are several parameters that govern the positional disorder on the atomic and the microscopic scales such as the position of the counterion, the inter-chain distance, the configuration of the polymer chains, the dimension of crystalline/amorphous domains (defined as the structural coherence length $\mathrm{L}_{\text {struc }}$ ), the orientation of crystalline domains. The wavefunction of the charge carrier tends to be spatially localized by the disorder, this is the so-called Anderson localization ${ }^{57}$. The charge carrier is characterized by a localization length Lloc, which can for instance represent the size of a polaron on one chain or the extension of its wavefunction over stacked adjacent chains. When the crystalline domains are larger than the spatial extent of the carrier $\left(\mathrm{L}_{\text {struc }}>\mathrm{L}_{\mathrm{loc}}\right.$ ), the disorder can be classified as inhomogeneous disorder. Conducting polymers in that situation can be seen as "granular metals" composed of metallic domains surrounded by an amorphous matrix limiting the conductivity $^{58}$ 59. The transport mechanism through the amorphous shell is crucial to understanding the transport in macroscopic samples ${ }^{60}$. If $\mathrm{L}_{\text {struc }}<\mathrm{L}_{\mathrm{loc}}$, the polymer sample has a homogeneous disorder ${ }^{61}$, that is to say, the average electronic coupling or transfer integral $t$ between the chains becomes the main parameter governing the interchain transport, and a three-dimensional transport is enabled in a sample comprising aggregated one-dimensional polymer chains ${ }^{62} 63$.

The (bi)polarons wavefunctions overlap on the same polymer chain forms an intra-chain band, while an inter-chain band is created by the interaction of (bi)polarons on adjacent chains. The Fermi level intrinsically lies within a polaronic band but can be also located within a broad bipolaronic band that overlaps with the valence band of the neutral polymer. It's been shown by Mott that for a disordered system the states in the middle of a band are extended states, while those lying in the tails are localized ${ }^{64,65}$. The separation between those two types of states takes place at an energy called the mobility edge $E_{c}$ (Fig. 8). High disorder leads to a long tail, while strong inter-chain coupling creates a broad region of extended states. The electrical conductivity of a conducting polymer with homogeneous disorder depends on the position of the Fermi level with respect to the mobility edge. If $\mathrm{E}_{\mathrm{F}}$ lies in the region of the extended states, the charge carrier has a large localization length, and the conductivity is metallic: $\sigma$ increases when cooling down and reaches a finite value at $\mathrm{T}=0$. In disordered sample, most of the states in the band are localized. $\mathrm{E}_{\mathrm{F}}$ within localized states implies that the carrier is localized and temperature activated hopping is needed for the transport between localized states. In such situation, $\sigma \rightarrow 0$, when $T \rightarrow 0$. Hence, a metallic behavior is expected for a polaron network since $E_{F}$ is in the middle of the polaronic band composed of extended states, as in the case of polyaniline ${ }^{42}$. Such metallic behavior is less likely to be observed for a bipolaron network, since $\mathrm{E}_{\mathrm{F}}$ is found between the valence band and the bipolaron band, where most of the states are localized. Such a system is called a Fermi glass. 


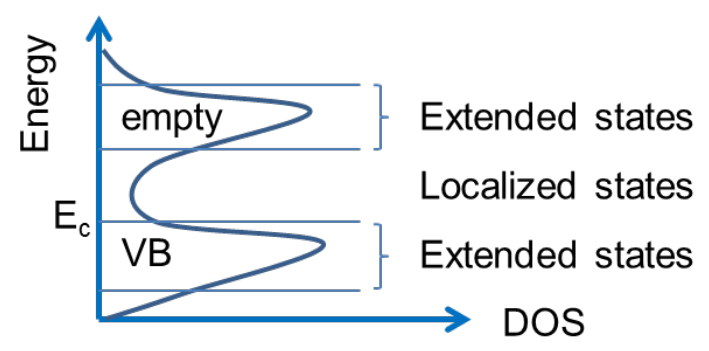

Fig. 8: Density of states of a disordered (oxidized) conducting polymer including extended and localized states close to the mobility edge $\mathrm{E}_{\mathrm{c}}$. The valence band is sketched close to an empty or half-filled band that can originate from bipolaron bands or polaron bands in case of highly doped conducting polymers.

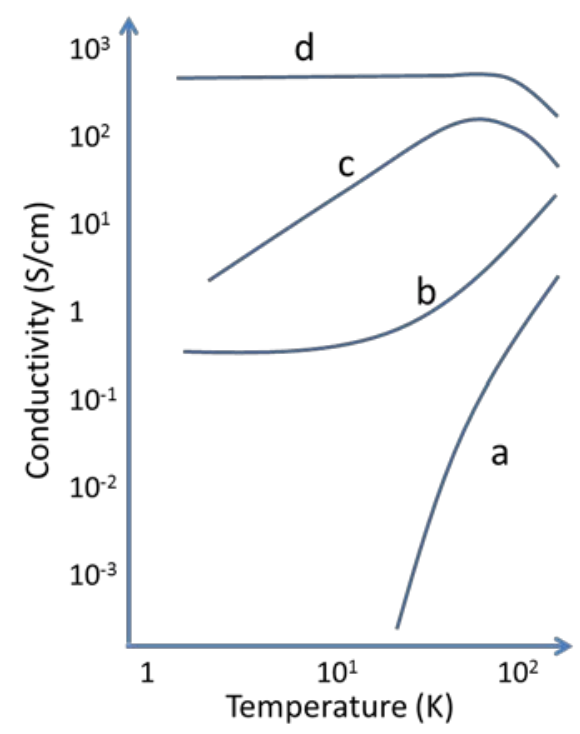

Fig. 9: Different cases of the electrical conductivity temperature dependence in conducting polymers.

Hence depending on the degree of disorder (energy-spatial), conducting polymers display several types of temperature dependence of the electrical conductivity. For disordered samples, the conductivity is low and increases when the temperature is raised. Conducting polymers behave like insulators with high activation energy $(<0.1 \mathrm{eV})$ and positive temperature coefficients (Curve a, Fig. 9). Lowering the degree of disorder prompts the electrical conductivity increase and the activation energy reduction (curve b). The charge transport in doped and disordered polymers is described by the hopping mechanism. Both molecular bipolarons and polarons can be transported in a thermally assisted hopping process. Above a small fraction of temperatures characterizing molecular vibrations polaron-hopping processes follow an Arrhenius-like behavior. The corresponding jump rates and activation energies can then be calculated classically. Bipolarons can be transported in a bipolaron breaking process that leads to a formation of two polarons that can hop independently from one another. Once the hop is completed the polarons can recombine again into a bipolaron ${ }^{47}$. The electrical conductivity for nearest- 
neighbour hopping is then proportional to the concentration of polarons $c_{p}$ and to the polaron hopping rate $R_{p}$.

$$
\sigma \cong \frac{N q^{2} a^{2}}{k T} c_{p} R_{p}
$$

Here $N$ is the density of sites between which polarons of charge $q$ move and $a$ is their characteristic hopping distance, $N a^{2} \sim 1 / a$. The semiclassical polaron jump rate can be written as:

$$
R_{p}=v e^{-\left(\frac{E_{p}}{2}-t\right) / k T} P
$$

where $v$ is the frequency characterizing the vibrations with which the carrier interacts, $t$ is the electronic transfer energy linking the sites between which the polaron hops, $E_{p}$ is the polaron binding energy, and $P$ is the probability that the electron carrier will hop between sites when vibrations establish a transient coincidence between their electronic energies. When the electronic transfer energy $t$ is large enough (comparable to the vibration energy $h v, P \approx 1$ ) a charge carrier is able to adiabatically follow atomic vibrations and thereby avail itself of every opportunity to hop ${ }^{66}$. Alternatively, when the electron transfer energy is very small, hopping becomes non-adiabatic with $P<<1$. Then $P$ is proportional to the square of the electronic transfer energy, which governs a carrier's hop. Note that in an undoped or lightly doped conjugated polymer, the polaron hops to a neutral adjacent segment under the influence of the electric field. In the doped disordered conjugated polymer, due to the high density of doping charges, i.e. a polaron associated with a counterion, the charge carriers can hop from one polaronic site to another without any systematic change in the structure of the segment constituting the hopping sites. In that case, the electron-phonon coupling is negligible and one transport parameter, namely the polaron binding energy or reorganization energy, can be neglected and replaced by the hopping activation energy. The conductivity can be described by hopping models between localized states constituting a density of state of a specific shape. The simplest approach postulates a constant density of state, as in the case of Mott's variable range hopping (VRH) or Efros-Shklovskii model ${ }^{67}$. This crude assumption is useful to grasp the essence of some important transport parameters. The conductivity dependence on $\mathrm{T}$ is expressed as 68 :

$\sigma(\mathrm{T})=\sigma(\mathrm{T}=0) \exp \left[-\left(\mathrm{T}_{0} / \mathrm{T}\right)^{\gamma}\right]$

where $\gamma$ is a dimensionality parameter ( $1 / 4$ for $3 \mathrm{D}$ system) that depends on the disorder extent as well as microstructure and morphology of the polymer; $\mathrm{T}_{0}$ is defined by the localization length $L_{l o c}$ and the density of states at the Fermi level $N\left(E_{F}\right)$ :

$\mathrm{L}_{\text {loc }}=\left[18 /\left(\mathrm{kT}_{0} \mathrm{~N}\left(\mathrm{E}_{\mathrm{F}}\right)\right)\right]^{1 / 3}$ 
Samples with a certain degree of structural order can display insulator-to-metal transitions. At first, upon cooling, the electrical conductivity diminishes but then stabilizes and remains constant below a specific temperature. This is so-called critical regime characterizing this transition (Curve b). The insulator-to-metal transition occurs as a consequence of the improved electronic coupling between the polymer chains with low vibrational amplitude, or, more specifically, due to an increase of the inter-chain localization length of the carriers. Highly doped and ordered conducting polymers, such as polyaniline with camphor sulfonic acid, show a truly metallic behavior at room temperature with an electrical conductivity that increases upon cooling down to $\sim 100 \mathrm{~K}^{42}$. Below $\sim 100 \mathrm{~K}$, the conductivity can be constant like in metals (curve d) or decrease (curve c). A conductivity drop when cooling the sample indicates yet another transition, this time from metallic to insulating regime. Although the origin of this effect is not fully understood, some explanations have been proposed within a model that considers 3D metallic regions electronically coupled via 1D chains that undergo a decrease of the localization length of the carriers when the temperature decreases ${ }^{69} 70$.

\subsection{Seebeck coefficient}

The thermodynamic definition of the Peltier coefficient is:

$\pi=\left(\frac{d Q}{d t} / \frac{d|e|}{d t}\right)_{\nabla T=0}$

which is the heat flow transported by a current when there is no temperature gradient applied. From that definition and using Kelvin's relation, the Seebeck coefficient is:

$\alpha=\left(\frac{d S}{d t} / \frac{d|e|}{d t}\right)_{\nabla T=0}=|e|\left(\frac{J_{S}}{J_{e}}\right)_{\nabla T=0}$

where $\mathrm{dS} / \mathrm{dt}=\mathrm{J}_{\mathrm{S}}$ is the flow of entropy $\mathrm{S}=\Delta \mathrm{Q} / \mathrm{T}$ and $\left(|\mathrm{e}| \mathrm{d} \mid \mathrm{e} / / \mathrm{dt}=\mathrm{J}_{\mathrm{e}}\right)$ is the electrical current. The Seebeck coefficient is proportional to the flow of entropy transported by a current when no temperature gradient exists. Depending on the type of solids and charge carriers, there exists an electronic contribution to the entropy flow $\mathrm{J}_{S}{ }^{\text {el }}$ and a phonon (vibrational) contribution $\mathrm{J}_{\mathrm{S}}{ }^{\mathrm{ph}}$, which are likely coupled via electron-phonon interactions or scatterings $\mathrm{J}_{\mathrm{S}}{ }^{\text {el-ph }}$. The Seebeck coefficient is expected to have three origins: the electronic Seebeck coefficient $\alpha^{\mathrm{el}}$, the phonon Seebeck coefficient $\alpha^{\mathrm{ph}}$ and the electron-phonon coupling coefficient $\alpha^{\text {el-ph }}$.

$\alpha=-|e|\left(\frac{J_{S}}{J_{e}}\right)_{\nabla T=0}=\sim\left(\frac{J_{S}^{e l}+J_{S}^{p h}+J_{S}^{e l-p h}}{J_{e}}\right)_{\nabla T=0}=\alpha^{e l}+\alpha^{p h}+\alpha^{e l-p h}$

The phonon contribution to the Seebeck coefficient, also called phonon-drag, is only distinct in crystalline materials (metal or semiconductor) at rather low temperatures $(<200 \mathrm{~K})$ when the phonon mean free path becomes large ${ }^{71}$. At high temperature, phononphonon scattering dominates minimizing the phonon mean free path, such that the 
phonon-drag effect is negligible. In crystalline conducting solids, when the phonon mean free path is of the same order of magnitude as the electron mean free path, the electronphonon scattering is significant. This contribution is expected at low temperatures for highly conducting polymers with significant degree of crystallinity ${ }^{72}$. In the insulating regime of conducting polymers, i.e. for significant energy and positional disorder, the phonon mean free paths is never large and almost reduced to the inter-chain distance. Hence, the charge transport proceeds through the temperature activated hopping. A contribution to $\alpha^{\mathrm{el}-\mathrm{ph}}$ originates from the coupling between the vibrational entropy upon charge carrier hopping ${ }^{73}$. Although not exactly introduced as proposed in Eq. 2.4.3, the later contribution has been proposed by Heikes ${ }^{74}$ and D. Emin ${ }^{75}$. This contribution of $\alpha^{\text {el- }}$ ${ }^{\mathrm{ph}}$ is called vibrational softening by $\mathrm{D}$. Emin and has been estimated to be up to $260 \mu \mathrm{V} / \mathrm{K}$ for pentacene an undoped conjugated organic semiconductor ${ }^{76}$. The charge carrier hopping on a neutral conjugated chain modifies the covalent bonding scheme, typically characterized by an inversion of the bond-length alternation in order to create a polaron: double $\mathrm{C}=\mathrm{C}$ bonds become single $\mathrm{C}-\mathrm{C}$ (softening) and single bond acquires a double bond character (hardening). This modification in the frequency of vibration $\Delta \mathrm{V}_{\mathrm{j}}$ is similar to the change in vibrational spectrum of a conjugated polymer upon doping; which is detectable by vibrational infrared (FTIR) and Raman spectroscopies ${ }^{77}$. When phonons with energies lower than $k T$ are involved, the vibrational entropy $\mathrm{S}_{\text {vib }}$ leads to a temperatureindependent contribution given $\mathrm{by}^{73}$ :

$$
\alpha_{v i b}^{e l-p h}=\frac{\Delta S_{v i b}}{q}=\frac{k}{q}\left(\sum_{j} N_{j}\left|\frac{\Delta v}{v}\right|_{j}\right)
$$

where $N_{j}$ represents the number of softened (or hardened) modes and $\vee_{j}$ their frequency, $\mathrm{q}$ is the charge of the carrier ( $q=+1|e|$ for positive polaron).

In the following part of this section, we will mostly focus on the electronic Seebeck contribution. In the case of a free electron gas there is only an electronic contribution $\alpha_{\mathrm{m}}{ }^{\mathrm{el}}$. The electrons at the hot side have a larger average energy than the electrons at the cold side, such that the difference in the average energies of both sides is $\mathrm{e} \Delta \mathrm{V}$; where $\Delta \mathrm{V}$ is the voltage difference induced by the temperature gradient. It follows that the Seebeck coefficient is linearly proportional to the temperature (curve a, Fig. 10):

$$
\alpha_{m}^{e l}=-\frac{\pi^{2} k^{2} T}{2 e E_{F(T=0)}}
$$

Another way to understand this phenomenon is to consider it in terms of thermoinduced diffusion effect of electrons. At the hot side, the mean free path of the electrons is larger than that at the cold side, such that in average more electrons are present at the cold side, and it leads to an electric potential difference. It means that the sign of the potential at the cold side tells us about the sign of the majority charge carrier. In case of metal, both holes and electrons thermo-diffuse and the resulting voltage is small, usually few $\mu \mathrm{V} / \mathrm{K}$. To explain that some metals have a positive Seebeck coefficient, it is necessary to 
consider electron scattering phenomena and the details of their band structure. The Seebeck coefficient of a real metal is still linearly proportional to the temperature (Eq. 2.4.6) and expressed in terms of the differential conductivity $\sigma(E)$, which is the conductivity that the system would have provided that the Fermi level were at energy $\mathrm{E}^{78}$ :

$\alpha_{m}^{e l}=\frac{\pi^{2}}{3}\left(\frac{k_{B}}{e}\right) k_{B} T\left[\frac{d \ln \sigma(E)}{d E}\right]_{E_{F}}$

While highly p-doped conducting polymers display very different electrical conductivity behaviors (Fig. 10), their Seebeck coefficients are typically positive and small $(<14 \mu \mathrm{V} / \mathrm{K}$ at $300 \mathrm{~K})$. This indicates that the majority charge carriers are positive carriers and that the metallic band is narrow ${ }^{79}{ }^{80}$. Therefore, the Seebeck coefficient decreases almost linearly with the temperature down to $10 \mathrm{~K}$ in a metallic manner (curve a in Fig. 10). This behavior was experimentally observed for polyacetylene ${ }^{81}$, polypyrrole ${ }^{82}$ and polyaniline $e^{83}$ and some other conducting polymers. In most of the samples the deviation from linearity is observed at very low temperatures indicating a strong electronphonon interaction in this region ${ }^{84}$ (curve b, Fig. 10) in absence of phonon drag. The latter normally manifests in a characteristic hump that disappears towards elevated temperatures (curve c, Fig. 10). Sometimes, upon cooling, the electrical conductivity can display a metal-to-insulator transition (curve c Fig 9), e.g. for polyaniline ${ }^{80}$, or an insulator-to-metal transition ${ }^{85}$ (curve b, Fig 9), but the Seebeck coefficient maintains a quasi-linear evolution (curves a, b, Fig. 10). This observation as well as the anisotropy of the Seebeck coefficient in stretch aligned films can be explained by the heterogeneous nature of conducting polymers ${ }^{86} 87,88$. The Seebeck coefficient is not so sensitive to the details of the bulk electronic conduction mechanism since the heat flow carried by phonons is not limited by insulating shells separating the metallic regions as much as the electric current 89 .

For lightly doped polymers, the Seebeck coefficient is larger than that for highly doped conducting polymers. Moreover, the temperature dependence of the thermopower is not linear anymore. The Seebeck coefficient can either decrease (curve d e in Fig 10) or increase (curve f) when the temperature drops. The non-linear decrease of $\alpha(T)$ is sometimes attributed to the $\mathrm{T}^{1 / 2}$-dependence obtained with Mott's variable range hopping transport between localized states (curve d):

$$
\alpha_{\text {hop }}^{e l}=\left.\frac{k_{B}^{2}\left(T_{0} T\right)^{1 / 2}}{2 e} \frac{\partial \ln N(E)}{\partial E}\right|_{E_{F}}
$$

where $\mathrm{N}(\mathrm{E})$ is the density of states. A decrease in doping level, leads to an increase in the Seebeck value (up to several hundreds of $\mu \mathrm{V} / \mathrm{K}$ ) ${ }^{37}$, but also a modification in the temperature evolution going from a linear $\mathrm{T}$ dependence to a $\mathrm{T}^{1 / 2}$-dependence (curve $\mathrm{d}$ ) ${ }^{61}$. When the Fermi energy lies in a region of localized states, there is a metallic contribution and a hopping contribution to $\alpha$, such that $\alpha(\mathrm{T})$ can be fitted experimentally by a $\mathrm{T}$ dependence and a $\mathrm{T}^{1 / 2}$-dependence: $\alpha(\mathrm{T})=\mathrm{AT}+\mathrm{BT}^{1 / 2}+\mathrm{C}$. The ratio of the two contributions depends on the temperature and the localization length of the carriers: 
$\left|\frac{\alpha_{h o p}^{e l}}{\alpha_{m}^{e l}}\right|=\left[\frac{T_{0}}{T}\right]^{1 / 2}$

If metallic contribution to thermopower dominates $T_{0}$ is usually very small indicative of weaker localization due to intrachain and interchain improved packing whereas for hopping regime $\mathrm{T} \ll \mathrm{T}_{0}$.

Hence, while the VRH could be an appropriate model to explain the decrease of the thermopower upon cooling, it fails in explaining the opposite trends $\alpha=\mathrm{f}(1 / \mathrm{T})$ observed for some conducting polymers (curve $\mathrm{f}$ ). $\alpha(\mathrm{T})$ designated by a negative slope is found in some conducting polymers on the insulating side of M-I transition, for example, in poly(estersyloxane)urethane ${ }^{90}$ or polythiophene ${ }^{91}$ doped with $\mathrm{I}_{2}$. Note that a $1 / \mathrm{T}$-dependence is typically observed in crystalline inorganic semiconductors when the carriers are transported in bands, but such a mechanism cannot be applied to these conducting polymers. This behavior can be attributed to so-called nearest-neighbor hopping thermopower $\alpha^{\mathrm{el}}$ nnh with $\mathrm{E}_{\mathrm{F}}$ within localized states (curve f) ${ }^{92}$ :

$\alpha_{n n h}^{e l}=\frac{1}{q} \frac{E_{A}}{T}+A$

where $\mathrm{q}$ is the charge, $\mathrm{E}_{\mathrm{A}}$ is activation energy and $\mathrm{A}$ is temperature independent kinetic term. More sophisticated model indicates that in a nearest neighbor hopping regime for a Fermi glass, both $\mathrm{T}$ - and 1/T-dependence can be obtained ${ }^{93}$. The hopping thermopower gradually decreases with raising temperature for low doping level sample and becomes nearly independent on $\mathrm{T}$ at higher doping levels (curve g).

Finally, we consider the effect of doping (change in a charge carrier concentration) on the thermopower of conducting polymers. In highly crystalline narrowband semiconductors characterized by polaron hopping, the localized polaronic states can be considered as almost degenerate with an energy distribution within kT (Fig. 11 left). In this limit, the Seebeck coefficient can be expressed directly on the basis of electronic entropy consideration and simple statistics of occupancy of the polaronic levels, which results in the Heikes formula ${ }^{94}$ :

$$
\alpha=\frac{k}{q} \ln \left(\frac{c}{1-c}\right)
$$

where $c$ is the fractional concentration of polarons $(c=n / N$, n charge carriers that occupy $\mathrm{N}$ degenerate levels) or the semiconductor's doping level. The intrinsic disorder in conducting polymers leads to polaronic levels spread over an energy range larger than kT, such that the Heikes formula is not valid anymore. Instead, the Seebeck coefficient must be expressed from the weighted average of the difference between the Fermi level and the energy $\varepsilon$ of the localized states contributing to the conductivity by $\sigma(\varepsilon)$ (Peltier energy $\Pi)$ : 


$$
\alpha=\left(\frac{k}{q}\right) \frac{\Pi}{k T}=\left(\frac{k}{q}\right) \frac{\int \sigma(\varepsilon)\left[\varepsilon-\varepsilon_{F}\right] d \varepsilon}{k T \int \sigma(\varepsilon) d \varepsilon}=\left(\frac{k}{q}\right) \frac{\left\langle\varepsilon-\varepsilon_{F}\right\rangle}{k T}
$$

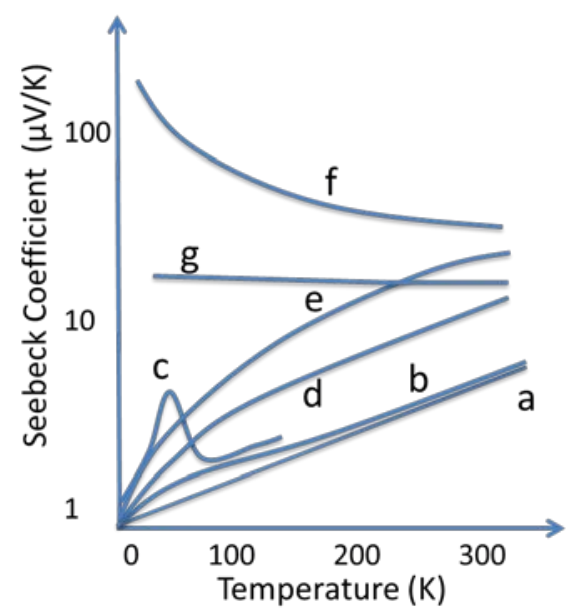

Fig. 10: Temperature dependence of the Seebeck coefficient for different types of conduction mechanism in conducting polymers.

If the localized states involved in the transport are distributed in a Gaussian density of states (DOS) ${ }^{95}$ (fig 11 right), the increase of charge carrier concentration is accompanied by displacement of the Fermi level in the DOS. As a first approximation, the middle of the Gaussian where the DOS is highest represents the transport level and the Seebeck coefficient is directly related to the difference in energy between the Fermi level and the transport levelThe thermopower is expected to vanish when the Fermi level is at the maximum of the DOS. In case of a perfectly symmetric DOS, and the Seebeck should change sign at 50\% doping level like in Heikes'formula.

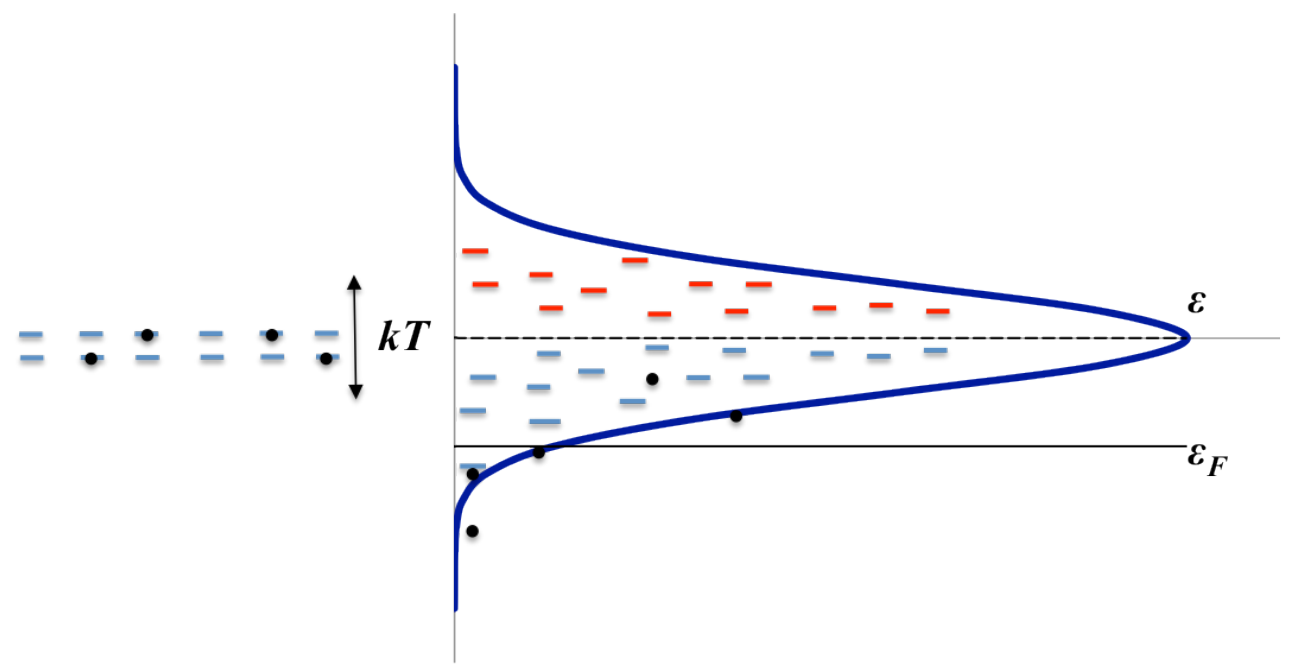

Fig. 11: Charge carrier distribution in Heikes formula limit with n charge carriers distributed over $\mathrm{N}$ available states (left); Gaussian density of states progressively filled upon doping, the thermopower changes sign when $\varepsilon_{\mathrm{F}}$ overpasses the midpoint of the DOS curve above $\varepsilon$ (right). 


\subsection{Thermal conductivity}

Only few studies report the thermal conductivity in conducting polymers. Typically, the values of thermal conductivity lies in the range of $0.1-0.7 \mathrm{Wm}^{-1} \mathrm{~K}^{-1}$ as for amorphous solids 969798 . Hence, the thermal conductivity of conducting polymers is not expected to demonstrate significant variations; however, even a small decrease matters a lot in terms of thermoelectric efficiency ZT. The total thermal conductivity of thermoelectric materials is the sum of an electronic contribution $\kappa_{\mathrm{e}}$ (charge carriers) and a lattice contribution $\kappa_{\mathrm{L}}$ (phonons). In order to estimate an upper limit for the electronic contribution, one can assume the ideal electron gas model (efficient heat transport) and use the Wiedemann-Franz law:

$\kappa_{e}=L T \sigma$

where $L$ is Lorentz factor ( $L=2.4 \times 10^{-8} \mathrm{~J}^{2} \mathrm{~K}^{-2} \mathrm{C}^{-2}$ ) and $\sigma=300 \mathrm{~S} / \mathrm{cm}$ typical of conducting polymers. This simple calculation shows that $\kappa_{\mathrm{e}}=0.002 \mathrm{Wm}^{-1} \mathrm{~K}^{-1}$ being a very small contribution that lies in the experimental detection limits. Note that the Wiedemann-Franz law breaks down for (bi)polaron charge carriers, such that an effective Lorentz number deviates from the value of a free electron gas and unusual temperature dependences of $\mathrm{L}$ appear $^{99}$. The small contribution of the charge carrier $\kappa_{\mathrm{e}}$ is also indicated by a study on polyaniline, which reveals that the thermal conductivity does not systematically vary with the electrical conductivity, despite the latter changes within nine orders of magnitudes ${ }^{96}$. Hence, the thermal conductivity of conducting polymers is dominated by the phonon transport and can be affected by dopants that may alter the heat capacity and the density of a polymer.

As previously mentioned, conducting polymers can be either amorphous or semicrystalline materials. No systematic studies have been conducted on the thermal conductivity of conducting polymer with an emphasis on the degrees of crystallinity. However, it's likely that conducting polymers display similar trends as non-conducting polymers. Thus we herein review briefly the main trends observed in non-conducting polymers, for it can serve as a guideline for conducting polymer based thermoelectrics.

The thermal conductivity of amorphous solid is not well understood and differs very much from that of a crystal. Surprisingly, the thermal conductivity in amorphous solids depends very little on the structure and chemical composition. Notably, for many dissimilar materials such as $\mathrm{SiO}_{2}$, Se, disorder crystals and polymers the magnitude of thermal conductivities at room temperature ${ }^{100}$ is comparable. Moreover, for amorphous materials the temperature dependence of the thermal conductivity generally displays the same trend (curve a in Fig. 11). There are three characteristic temperature intervals: (i) $\mathrm{T}<1 \mathrm{~K}$ where $\lambda(\mathrm{T}) \sim \mathrm{T}^{2}$; (ii) $\mathrm{T} \approx 10 \mathrm{~K}$, where the thermal conductivity is constant (plateau); and (iii) high temperature ( $\mathrm{T}>10 \mathrm{~K})$, where $\lambda$ rises smoothly to a limiting value given by the minimum thermal conductivity equation ${ }^{101}$ : 
where $\mathrm{L}$ is the phonon mean free path, given by the smallest distance in the solid: interatomic distance or interchain distance for polymer, $v$ is the velocity of sound and $\mathrm{C}$ is the classical specific heat. Nowadays, with the help of supercomputers and molecular dynamics software capable of modeling the disorder and the anharmonicity of the vibrational potential, these universal trends in amorphous solid become reproducible $\mathrm{e}^{102}$ 103 .

The thermal conductivity of semicrystalline polymers exhibits temperature dependence vastly different from that of the amorphous counterparts (this typical behavior is illustrated by curves b and c in Fig.11). No plateau region is observed around $10 \mathrm{~K}$. Instead the thermal conductivity increases as a power law $\mathrm{T}^{\mathrm{n}}$, with $\mathrm{n}$ varying between 1 and 3 below 20K. For polymers of comparatively low crystallinity, the thermal conductivity increases monotonically up to their respective glass transition temperatures (curve b). In contrast, for the highly crystalline (volume fraction crystallinity $>0.7$ ) polymers, the thermal conductivity is high and first increases with increasing temperature, reaches a peak near $100 \mathrm{~K}$ and subsequently drops (curve c) ${ }^{104}{ }^{105}$. In organic crystals, the thermal conductivity can reach up to $2 \mathrm{Wm}^{-1} \mathrm{~K}^{-1}$ at room temperature ${ }^{106}$ with a peak at low temperatures that is typical for crystalline materials (curve d). The thermal conductivity linearly decreases from room temperature down to $100 \mathrm{~K}$. Below that, the thermal conductivity increases to a maximum at about 20K. This behavior, commonly observed in semi-crystalline and crystalline organic materials, is attributed to the attenuation of the phonon-phonon scattering promoting large phonon mean free path $\mathrm{L}$ and enhancing the thermal conductivity. Below 20K, $\kappa$ drops abruptly likely due to a combination of decrease in heat capacity C (Debye model) and other phonon scattering phenomena.

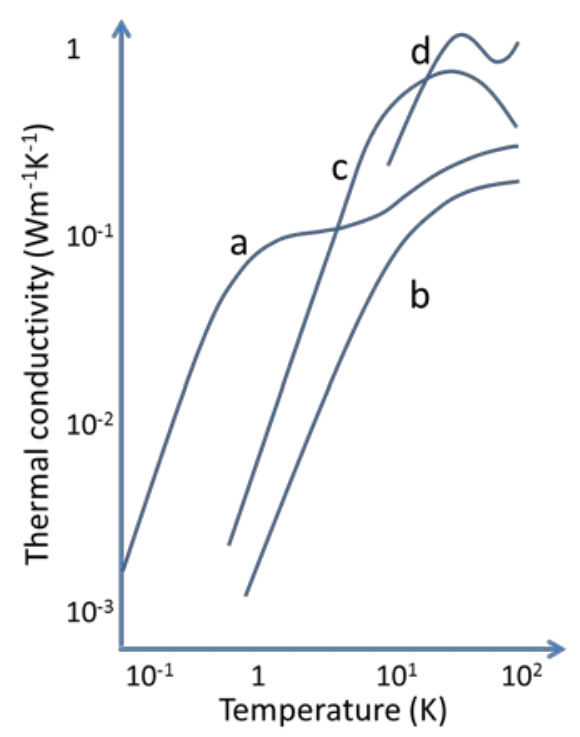

Fig. 12: Possible evolution of the thermal conductivity versus temperature for various types of organic materials. 
Finally, the thermal conductivity can be anisotropic since polymer chains can be aligned macroscopically due to the fabrication method. For instance, ratio of thermal conductivity measured parallel and perpendicular to a polymer film $\kappa / / / \kappa \perp$ can be as large as 4 for spin-coated polyimide films ${ }^{107}$ and extruded polyethylene can reach an anisotropy up to $10^{108}$.

\section{Polymer-based thermoelectric materials}

\subsection{Thermoelectric performance of pure conducting polymers (CPs)}

The expression for the dimensionless figure-of-merit ZT, which represents an ultimate measure of the thermoelectric efficiency, is given by:

$Z T=S^{2} \sigma T / \kappa \quad$ (3.1.1.),

where $S, \sigma$ and $\kappa$ are thermopower, electrical conductivity and thermal conductivity respectively. Since the conducting polymers are not stable at high temperature, their maximum operational temperatures are limited to $200-250^{\circ} \mathrm{C}$. Hence the benchmark for polymer-based thermoelectrics are $\mathrm{Bi}_{2} \mathrm{Te}_{3}$ alloys that have a $\mathrm{ZT}$ of 1.2 at room $\mathrm{T}$ (1.4 at $\left.400^{\circ} \mathrm{K}\right)^{109}$. The thermal conductivity of bulk $\mathrm{Bi}_{2} \mathrm{Te}_{3}$ is $1.4 \mathrm{Wm}^{-1} \cdot \mathrm{K}^{-1}$ and can be reduced to $1.1 \mathrm{Wm}^{-1} \cdot \mathrm{K}^{-1}$ by the introduction of nanostructures; which is still four to five times higher than typical values of thermal conductivity for a conducting polymer ${ }^{6}$. The Seebeck coefficient of $\mathrm{Bi}_{2} \mathrm{Te}_{3}$ alloys is about $210 \mu \mathrm{V} / \mathrm{K}$ and the electrical conductivity about $10^{5} \mathrm{~S} / \mathrm{m}$, giving a power factor about $10^{-3} \mathrm{Wm}^{-1} \mathrm{~K}^{-2}$ at room temperature. The limitation of conducting polymers is their rather low power factors that are normally in the order of $10^{-}$ ${ }^{5}-10^{-8} \mathrm{Wm}^{-1} \mathrm{~K}^{-2}$. Polyacetylene is the only example of a conducting polymer exhibiting truly high thermoelectric performance with the highest power factor of $10^{-4} \mathrm{Wm}^{-1} \mathrm{~K}^{-2}$ mainly owning to its outstandingly high electrical conductivity ${ }^{110}$. The thermoelectrical properties of polyacetylene vary considerably with doping concentration. The power factor for different samples with the doping level from $0.8 \%$ up to $28 \%$ increases by three orders of magnitude, from $1.4 \times 10^{-7} \mathrm{Wm}^{-1} \mathrm{~K}^{-2}$ to $4 \times 10^{-4} \mathrm{Wm}^{-1} \mathrm{~K}^{-2} 111$. This enhancement originates from the rise in the electrical conductivity, since the Seebeck coefficient undergoes a sixfold decrease from $120 \mu \mathrm{V} / \mathrm{K}$ to $20 \mu \mathrm{V} / \mathrm{K}$ as the doping proceeds. Despite such an encouraging performance, aging and environmental stability issues excluded this polymer from the potential candidates for thermoelectric applications. In contrast to polyacetylene, polyaniline (Pani) is known as a polymer of exceptional stability and easy processing. Consequently, quite a few studies on its thermoelectric properties are available. The results provided by $\mathrm{N}$. Mateeva et al. suggest that maximum ZT of acid doped PANI is $10^{-5112}$. N. Toshima et al. have reported one order of magnitude higher ZT at room $\mathrm{T}$ improved by stretching of (CSA)-doped Pani and ZT=0.01 for a multilayered film composed of doped and insulating Pani layers ${ }^{113}$. Relatively high power factors have been measured for $\mathrm{FeCl}_{3}$-doped polycarbazole ${ }^{114}$ derivatives $\left(19 \mu \mathrm{Wm}^{-1} \mathrm{~K}^{-2}\right)$ and 
polyphenylenevinylene (PPV) derivatives doped with iodine $\left(30 \mu \mathrm{Wm}^{-1} \mathrm{~K}^{-2}\right)^{115}$. The electrical conductivity of the latter can be appreciably increased, while the Seebeck coefficient remains high (around $40 \mu \mathrm{V} / \mathrm{K}$ ) and stable at various stretching ratios. Finally, $\mathrm{N}$. Toshima et al. reported a $\mathrm{ZT}=0.1$ a room temperature for $\mathrm{I}_{2}$ doped PPV derivatives ${ }^{116}$. Recently Y. Sun et al have developed p- and n-type poly(metal 1,1,2,2ethenetetrathiolate)s highly stable materials suitable for thermoelectric applications ${ }^{117}$. The reported ZT values for n-type samples at $400 \mathrm{~K}$ are 0.1 and 0.2 for Poly[Nax(Ni-ett)] and Poly[Kx(Ni-ett)] respectively. The latter has an extremely high negative Seebeck coefficient of $-121.6 \mu \mathrm{V} \mathrm{K} \mathrm{K}^{-1}$ which along with reasonable electrical conductivity and low thermal conductivity yields the highest ZT among stable n-type organic materials.

The addition of a secondary dopant can foster the thermoelectric efficiency enhancement via morphological reorganization that ameliorates the charge carrier mobility. This approach fulfills the same goal as polymer stretching but does not imply any mechanical intervention offering a manufacturing advantage. Unlike the electrical conductivity, the Seebeck coefficient is fairly insensitive to the addition of the secondary dopants, i.e. to a morphology change. Secondary doping effect appears useful for ZT improvement. For example, in poly(3,4-ethylenedioxythiophene):poly(styrenesulfonate) (PEDOT:PSS) even a small amount of dimethyl sulfoxide (DMSO) or diethylene glycol (DEG), N,N-dimethylformamide (DMF) and tetrahydrofuran (THF) enables a phase segregation of the excess PSS $^{43}$ and eventually the transformation of a coil conformation of the polymer into a linear or expanded-coil conformation which favors a better packing of the chains ${ }^{118}$. Untreated samples of PEDOT:PSS have a very poor thermoelectric performance as a result of low electrical conductivity $(0.06 \mathrm{~S} / \mathrm{cm})$ and metallic small positive thermopower $(12 \mu \mathrm{V} / \mathrm{K})^{119}$. Followed by a progressive increase in the secondary dopant concentration, the power factor can be changed by almost four orders of magnitude ${ }^{120}$. The power factor saturation occurs at $5 \mathrm{wt} \%$ DEG and $10 \mathrm{wt} \%$ DMSO and the maximum reported ZT of PEDOT:PSS is $1.75 \times 10^{-3}$ near $270 \mathrm{~K}^{119}$.

When optimizing inorganic semiconductors for thermoelectric applications, the first strategy would be to tune the doping level to maximize the power factor ${ }^{7}$. Obviously, the increase of charge carrier concentration leads to an enhancement of the electrical conductivity $\sigma$ and a reduction of the Seebeck coefficient $\alpha$. Hence, there exists a doping level at which the power factor $\sigma \alpha^{2}$ is maximum. We have applied this strategy to a conjugated polymer poly(3-hexylthiophene) and doped it with $\mathrm{NOPF}_{6}$ to increase the power factor from $10^{-10}$ to $10^{-7} \mathrm{Wm}^{-1} \mathrm{~K}^{-2}$. The modest electrical conductivity $(\sim 1 \mathrm{~S} / \mathrm{cm})$ precludes the power factor being sufficiently high ${ }^{37}$. PEDOT-PSS undoped with the thermal decomposable ammonium formate was found to exhibit a power factor increase from $0.019 \mu \mathrm{Wm}^{-1} \mathrm{~K}^{2}$ to $0.69 \mu \mathrm{Wm}^{-1} \mathrm{~K}^{2}$, but this effect has less positive implications than the the secondary dopant effect (power factor gain up to $7 \mu \mathrm{Wm}^{-1} \mathrm{~K}^{2}$ ) ${ }^{121}$. Hence, a polymer with high electrical conductivity is a prerequisite for further doping optimization. For poly3,4-ethylenedioxythiophene-tosylate (PEDOT-Tos) with the conductivity about $300 \mathrm{~S} / \mathrm{cm}$, we were able to optimize the power factor by means of a gradual reduction of the p-doped polymer with tetrakis(dimethylamino)ethylene (TDAE) ${ }^{41}$. The conductivity experiences a dramatic change as it diminishes from $300 \mathrm{Scm}^{-1}$ at $36 \%$ oxidation level 
down to $10^{-4} \mathrm{Scm}^{-1}$ at $15 \%$ oxidation level (Fig. 12). The Seebeck coefficient $\alpha$ is modified by a factor of 20 upon exposing the polymer to the TDAE vapor. Initially, at $36 \%$ oxidation level $\alpha$ is about $40 \mu \mathrm{V} / \mathrm{K}$, it reaches its maximum at $780 \mu \mathrm{V} / \mathrm{K}$ at the lowest measured oxidation level. The power factor $\sigma \alpha^{2}$ increases by a factor of 6 , from 53 $\mu \mathrm{Wm}^{-1} \mathrm{~K}^{2}$ for the pristine doped polymer to $324 \mu \mathrm{Wm}^{-1} \mathrm{~K}^{2}$ at the oxidation level of $22 \%$. At this optimum oxidation level, ZT attains a value of 0.25 , which nearly fulfills a requirement for efficient energy conversion.

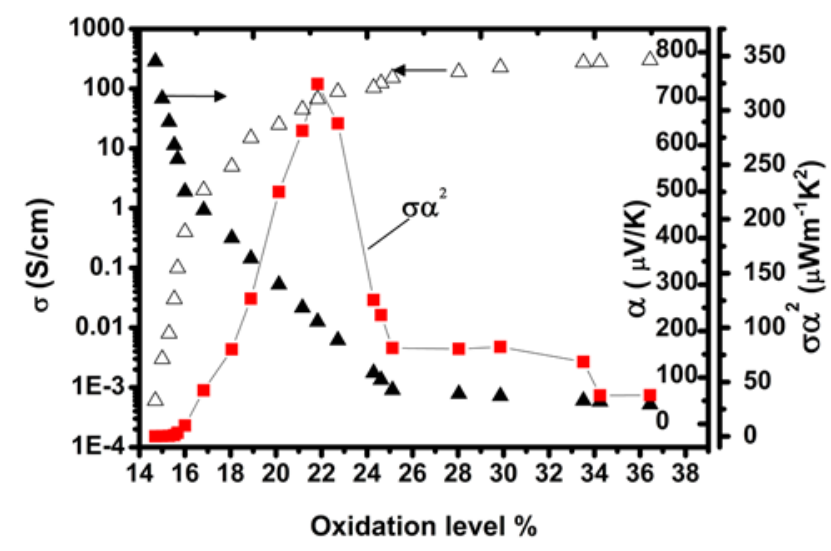

Fig. 13: Seebeck coefficient, electrical conductivity and corresponding power factor $\sigma \alpha^{2}$ of PEDOT-Tos vs. oxidation level. (reproduced with permission from ref ${ }^{41}$ )

\subsection{Conducting polymer composites using inorganic nanostructures}

Polymer based nanocomposites are envisioned as one of the most promising approaches to combine both the solution processibility of the conducting polymer and the good thermoelectric properties of the inorganic filler. Thus, a conducting polymer matrix could be used to lower the electric contact resistance between inorganic inclusions, simultaneously maintaining a low overall thermal conductivity of the composite. The low dimensionality thermoelectric materials possess intrinsically low thermal conductivity due to phonon interface scattering and therefore provide yet another alternative strategy ${ }^{122}$. In practice this approach does not imply high temperature treatment to sinter the inorganic nanoparticles.

Rod-shaped tellurium nanocrystals dispersed in a matrix of PEDOT:PSS yield ZT = 0.1 at $\mathrm{RT}^{123}$. This encouraging result is a result of a combination of an improved power factor $\sigma \alpha^{2}=70 \mu \mathrm{WK}^{-2} \mathrm{~m}^{-1}$ and a low thermal conductivity $\lambda=0.2 \mathrm{Wm}^{-1} \mathrm{~K}^{-1}$ (identical to the polymer). P-type [n-type] $\mathrm{Bi}_{2} \mathrm{Te}_{3}$ particles impregnated into the PEDOT:PSS(DMSO) emulsion create a composite with a power factor of $130 \mu \mathrm{Wm}^{-1} \mathrm{~K}^{-2}{ }^{124}$. Interestingly, the $10 \%$ of PEDOT:PSS (of low Seebeck coefficient) in the composite does not impair the negative Seebeck coefficient of the n-type $\mathrm{Bi}_{2} \mathrm{Te}_{3}$ particles, thus indicating an attractive route for n-type polymer composite formation. The estimation of ZT for $10 \%$ PEDOT:PSS and 90\% p-type $\mathrm{Bi}_{2} \mathrm{Te}_{3}$ is 0.08 and is likely underestimated since the 
assumed thermal conductivity for the composite does not include the effect of phonon scattering at the polymer-particle interface.

One of the issues with semi-metal or semiconducting nanostructures is their sensitivity to dioxygen (creation of an oxide layer), which is detrimental for the electrical conductivity of the polymer composites. In that context, carbon-based nanostructures (carbon nanotubes CNT, graphene) might offer an interesting alternative. A composite made of Gum Arabic insulating particles whose interstitial spaces are filled with 35\% CNT and 35\% PEDOT:PSS are characterized by ZT=0.02 at room temperature ${ }^{125}$. With the electrical conductivity of $400 \mathrm{~S} / \mathrm{cm}$ and the Seebeck of $26 \mu \mathrm{V} / \mathrm{K}$ the power factor reaches $25 \mu \mathrm{WK}^{-2} \mathrm{~m}^{-1}$. As for the thermal conductivity of the composite, its value $(0.36$ $\left.\mathrm{Wm}^{-1} \mathrm{~K}^{-1}\right)$ is much lower than the thermal conductivity of a CNT $\left(\sim 1000 \mathrm{Wm}^{-1} \mathrm{~K}^{-1}\right)$. This low thermal conductivity, for such a high CNT content, is attributed to the presence of PEDOT:PSS acting as thermal insulating barriers between the carbon nanotubes. Note however that insulating polymer-CNT composites display a similar thermal property but for a much lower CNT content $(\sim 1 \mathrm{wt} \%)^{126}$. A step further in controling the thermoelectric properties of CNT-polymer composite is to use a strong electron donor and acceptor to change the charge carrier concentration in CNT in a similar manner as it is done with conducting polymers ${ }^{127}$. For instance the addition of polyethyleneimine, a reducing agent ${ }^{128}$, with CNT leads to a composite with negative Seebeck coefficient $(-58 \mu \mathrm{V} / \mathrm{K})$; while the pristine Seebeck coefficient of CNT is $+28 \mu \mathrm{V} / \mathrm{K}$. This result is especially encouraging taking into account that stable n-type organic materials with good thermoelectric properties are not easy to obtain.

\section{Organic Thermoelectric generators}

\subsection{Theory of thermoelectric energy conversion}

In a thermocouple of internal resistance $\mathrm{R}$ subject to a temperature gradient $\Delta \mathrm{T}=\mathrm{T}_{\mathrm{H}^{-}}$ $\mathrm{T}_{\mathrm{C}}$, we assume that the heat propagating from the hot to the cold side can only pass through the thermoelectric legs and that the metal junction connecting the legs has a negligible electrical and thermal resistance. The thermal energy flow delivered at the hot junction is divided in three contributions (Fig. 13): (i) the rate of the heat passing through the leg $\mathrm{K} \Delta \mathrm{T}$ in absence of a generated electrical current ( $\mathrm{K}$ is the thermal conductance of the thermocouple); (ii) the Joule heat $1 / 2 \mathrm{I}^{2} \mathrm{R}$ provided to the hot side (opposite to the heat flow $\mathrm{K} \Delta \mathrm{T}$ ) originating from the generated thermoelectric current I due to the thermoinduced voltage $\mathrm{V}=\alpha \Delta \mathrm{T}$ (Joule heating heats the middle of the leg, so that one-half goes to each end of the leg); (iii) the rate of Peltier heat absorption $\alpha \mathrm{T}_{\mathrm{H}} \mathrm{I}$ from the hot side due to the thermoelectric electric current going to the cold side (same direction as the heat flow $\mathrm{K} \Delta \mathrm{T}$ ). The thermal power input to the hot junction is then:

$$
Q_{H}=\alpha T_{H} I-\frac{1}{2} R^{2}+K\left(T_{H}-T_{C}\right)
$$




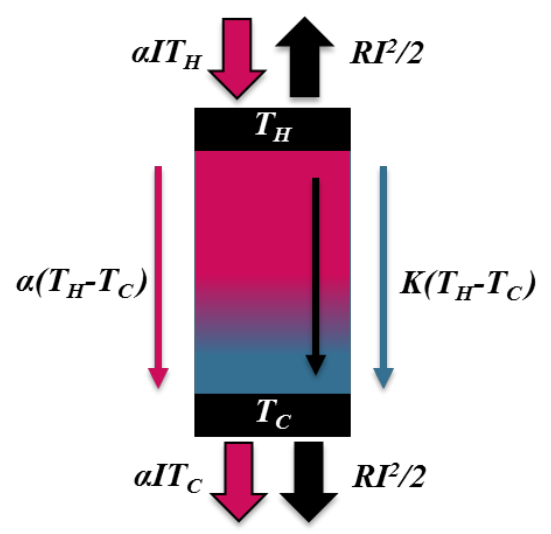

Fig. 14: Sketch of the different energy flows passing through a thermoelectric leg subjected to a temperature gradient.

When the thermocouple is coupled with a load resistance $\mathrm{R}_{\mathrm{L}}$, the electrical power output is:

$$
P=R_{L} I^{2}
$$

with a current:

$$
I=\alpha \Delta T /\left(R+R_{L}\right)
$$

and an open-circuit voltage:

$$
V=\alpha \Delta T
$$

The efficiency $\eta$ of the thermoelectric generator is the ratio between the thermal power input $\mathrm{Q}_{\mathrm{H}}$ and the electrical power output $\mathrm{P}$ :

$$
\eta=R_{L} I^{2} /\left(\alpha T_{H} I-1 / 2 I^{2} R+K \Delta T\right)
$$

The maximum efficiency $\eta_{\max }$ is obtained by matching the properties of the legs and the load resistance to the internal resistance (further reading see e.g. ${ }^{74}$ ). Assuming that the thermoelectric properties of materials $(\alpha, \kappa, \sigma)$ are constant with the temperature (never the case in reality), $\eta_{\max }$ is given by:

$$
\begin{gathered}
\eta_{\max }=\frac{T_{H}-T_{C}}{T_{H}} \frac{M-1}{M+T_{C} / T_{H}} \\
M=\left[1+\frac{1}{2} Z\left(T_{H}-T_{C}\right)\right]^{1 / 2}
\end{gathered}
$$

where $\mathrm{Z}$ is the figure of merit of a single leg or the combined figure of merit if the device 
contains both $\mathrm{p}$ - and $\mathrm{n}$-legs. In case of a thermocouple the expression for $\mathrm{Z}$ is given by:

$$
Z=\frac{\left(S_{a}-S_{b}\right)^{2}}{\left[\left(\rho_{a} \kappa_{a}\right)^{1 / 2}+\left[\left(\rho_{b} \kappa_{b}\right)^{1 / 2}\right]\right]^{2}}
$$

The evolution of the maximum efficiency versus temperature for various values of $\mathrm{Z}$ is plotted in Fig. 2. The first term $\left(\mathrm{T}_{\mathrm{H}}-\mathrm{T}_{\mathrm{C}}\right) / \mathrm{T}_{\mathrm{H}}$ in the equation (4.1.6) represents the reversible Carnot efficiency, while the second term stands for the irreversible component to the overall efficiency that denotes the deviation from the ideal heat engine performance. Strictly speaking, the efficiency is lowered by Joule heating and the irreversible losses of heat conduction. Additionally, the efficiency decrease further in a real TEG because of the losses on the electrical interconnects, the electrical and thermal contact resistances, etc.

In the regime of maximum power output, the TEG must be connected to an external load resistance equal to the internal resistance of the device $R_{L}=R$. The voltage output is half of the open circuit voltage $\mathrm{V}=\mathrm{V}_{\mathrm{oc}} / 2$ and the maximum generated power is:

$$
P_{\max }=\frac{V_{o c}}{4 R}=\frac{\alpha^{2}\left(T_{H}-T_{C}\right)^{2}}{4 R}
$$

The conditions for maximum power output and maximum conversion efficiency at fixed geometry of the legs are in mutual contradiction. The ratio between load resistance and internal resistance for maximum conversion efficiency is expressed by 4.1.5, whereas for maximum power output configuration two resistances must be equal. Therefore, a TEG can be operated in two different modes. At constant temperature gradient, the power output increases as the legs becomes shorter (low internal resistance) since the unfavorable Joule heating contribution is reduced (see Eq. 4.1.1). Now, changing the length of the leg allows setting various temperature gradients. To improve the TEG's maximum efficiency longer legs are required, so that eventually any thermoelectric module is designed under optimal conditions dictated by a trade-off between $P_{\max }$ and $\eta_{\max }$. Today's manufacturing technologies allow for smaller scale thermoelectric conversion, which is highly desirable from a power density point of view. Regardless of the size a small TEG can produce the same amount of power as a large one if they have equal proportions between the device area and its thickness. However, for micro-TEGs, the amount of heat supplied to a hot side is significantly reduced.

\subsection{Early OTEG prototypes}

The main advantages of organic TEGs over inorganic ones are essentially related to inexpensive manufacturing techniques, since conducting polymers can be processed from solution at low temperature. Hence the techniques, such as printing, electropolymerization, drop casting and others are possible. Additionally, polymer-based 
micro-thermoelectric generators could be fully flexible, which is a key feature for specific applications. Even though the main efforts in the domain of organic thermoelectrics are still aimed at improving further the material's efficiency, the first OTEG prototypes have already been demonstrated.

One of the first organic-based TEGs consisted of three screen-printed legs (length $=2 \mathrm{~cm}$, width $=0.5 \mathrm{~cm}$ and thickness $=70 \mu \mathrm{m}$ ). The p-leg was composed of a blend of polyvinylchloride (PVC) and graphite; the n-leg was made of PVC and the organic charge transfer salt TTF-TCNQ ${ }^{129}$. The thermovoltage of this 3-leg printed OTEG is $120 \mu \mathrm{V} / \mathrm{K}$. Because of the lateral architecture of the legs, the device is easily printed and large temperature gradient can be obtained. The major drawback is the large internal resistance $(\mathrm{R}=250 \mathrm{k} \Omega)$ limiting the power output. Hence a lateral architecture is likely useful to replicate large numbers of legs and achieve large voltage for temperature sensing applications, but not for power generation.

We made a second prototype of a fully organic TEG consisting of 55 legs in a vertical architecture $^{41}$. Each leg is about $1 \times 1.5 \mathrm{~mm}$ of base and $30 \mu \mathrm{m}$ in length. The legs are obtained by filling cavities with precursor solutions with micropipettes. The cavities are designed with a photoresist on top of the bottom Au electrodes (Fig. 14). The n-legs are composed of a blend of TTF-TCNQ with PVC; while the p-legs are composed of PEDOT-Tos created by direct chemical polymerization of EDOT. The maximum power output was $0.128 \mu \mathrm{W}$ at $\Delta \mathrm{T}=10 \mathrm{~K}$. The thickness of the device is limiting $\Delta \mathrm{T}$, so that higher power could be achieved with longer legs and higher packing density. We therefore extrapolated the expected power generated per unit area to $0.27 \mu \mathrm{W} \mathrm{cm}{ }^{-2}$ at $\Delta \mathrm{T}=30 \mathrm{~K}$. With the manufacturing technique used, this micro-OTEG could easily be composed of many more legs and be flexible.

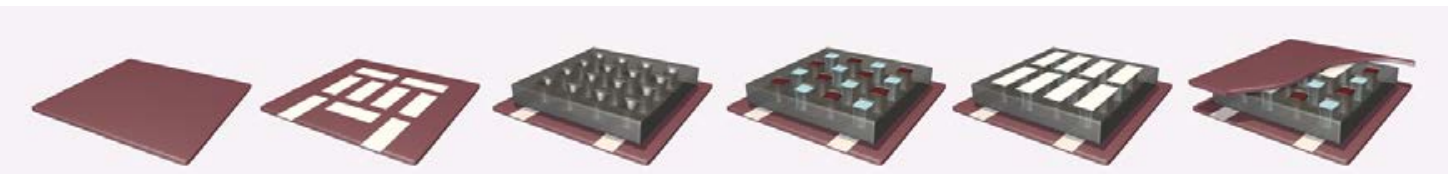

Fig. 15: Fabrication steps for the OTEG based on PEDOT-Tos/TTF-TCNQ.

The third OTEG prototype in vertical architecture was realized by Y. Sun et al. ${ }^{117}$. The fabrication technique was different in the sense that the metal-organic conducting polymers (poly(metal 1,1,2,2-ethenetetrathiolate) were insoluble. Hence, the legs were formed by pressing the polymer powder into pellets with the following dimension: $5 \times 2$ $\mathrm{mm}$ for the base and $0.9 \mathrm{~mm}$ for the length. This OTEG is thus not a microthermogenerator but a robust device for higher power generation. The top and bottom side of the pellets were coated with a thin Au layer in order to enable a better contact to the interconnects during the module assembly. The OTEG consisted of 35 legs and generated $\mathrm{V}_{\mathrm{oc}}=0.26 \mathrm{~V}$ for $\Delta \mathrm{T}=82 \mathrm{~K}$ (Fig. 15). Compared to our previous prototype (PEDOT-Tos /TTF-TCNQ), the power output is six time larger, reaching $1.2 \mu \mathrm{W} \mathrm{cm}{ }^{-2}$ at $\Delta \mathrm{T}=30 \mathrm{~K}$. 
Since the legs are much longer than in the previous prototype, the mechanical flexibility is likely difficult to achieve, however large $\Delta \mathrm{T}$ up to $80 \mathrm{~K}$ could be obtained providing a maximum power of $750 \mu \mathrm{W}$.
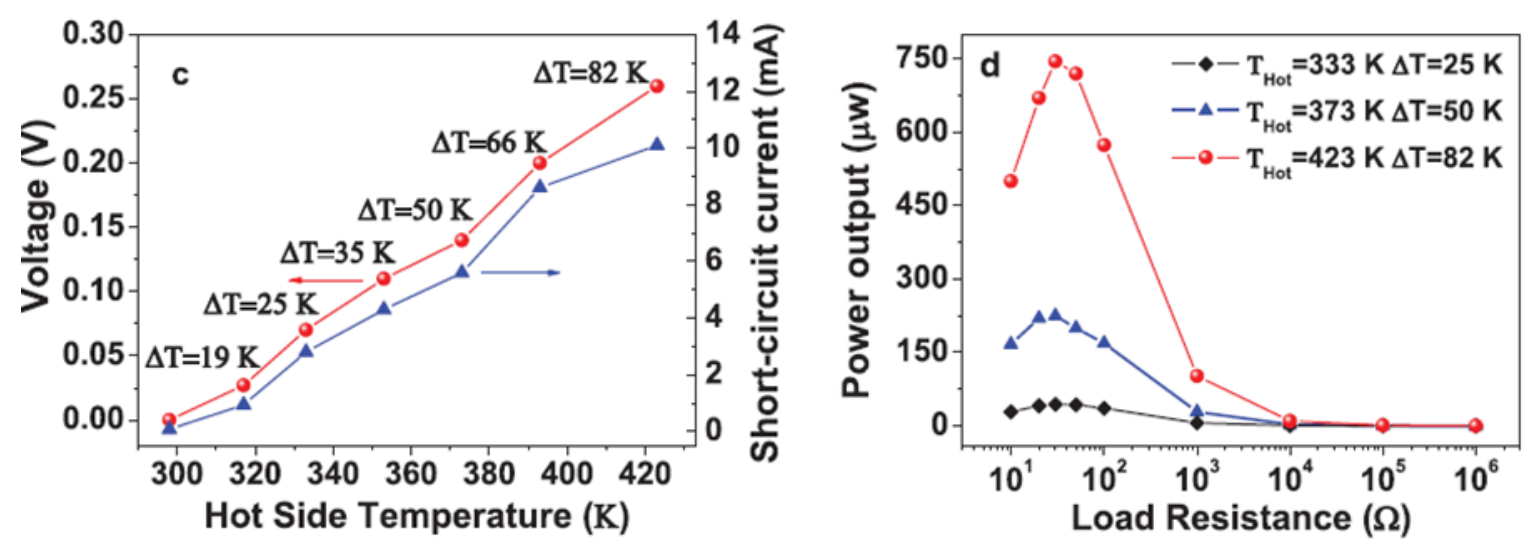

Fig. 16: Evolution of the open-circuit voltage versus $\Delta \mathrm{T}$ (right) and power output versus load resistance for three different $\Delta \mathrm{T}$. (reproduced with permission from ref ${ }^{117}$ )

Already with those early prototypes, OTEGs can potentially drive small electronic devices that usually require not more than 2V. OTEGs can charge supercapacitors able to release a peak power needed for communication technologies. The manufacturing process of miniaturized TEGs fabrication can largely avail of excellent processibity of CPs that can be patterned into thousands of thermocouples scaled down to a small area by printing, drop casting, or electrochemical deposition on rigid or flexible substrates. Furthermore, the restraints imposed on TEGs design are not dictated by the processing limitations but rather by TEGs functionality. These devices can operate at modest temperature gradients and still exhibit relatively high power generation densities sufficient for applications in autonomous microsystems or wearable electronics. Large area OTEGs for waste heat recovery should consist of multiple OTEG modules delivering reasonable voltage and connected in parallel to supply large current.

\section{Prospects}

We are yet to fully comprehend the potentials of organic thermoelectrics as it just begins to emerge as one of the alternatives for low temperature heat recovery. Even though the thermoelectric properties of conducting polymers are not fully understood, there are certainly some strong indications that the main problem for further ZT enhancement comes from a generally low power factor. Macromolecular conjugated systems are really complex in morphology with polymer chains coiling up, aggregating in some regions more than in the others, forming crystalline islands separated by amorphous inclusions. It can result in a set of thermoelectric properties, which are highly anisotropic and strictly dependent on synthesis and processing. Obviously, one way to improve the conductivity is to enhance their crystallinity by controlling their structure and surface 
morphology. The metallic conducting polymer is a special case that is of no much interest for thermoelectric applications since the corresponding thermopower is too low due to the low Seebeck coefficient. Thus the "insulating" regime close to the critical regime should be the focus of the thermoelectric research. In this regime, the transport is still slightly thermally activated with a localization length larger than in strongly disorded systems (low doping level and low crystalline ratio). The coupling between the conjugated chains via the wave-function of the counterion might be of crucial importance in the inter-chain transport, the effect which is barely studied ${ }^{130}$. Hence, the packing of the polymer chains and the nature and position of the counterions in the crystalline region appear essential for the electrical conductivity.

The Seebeck effect is little understood and only simple models have been proposed up to now. Since the optimization of the powerfactor is realized by tuning the doping level, it appears essential to refine the models describing the entropy-of-mixing contribution $\alpha^{\mathrm{el}}$ mix with a more realistic density of states (Eq. 2.4.10). Hence, similar to the optimization of bands in semiconductors ${ }^{131}$, the density-of-states can be designed to optimize $\alpha^{\mathrm{el}}$ mix. Note that from the simple expression of the Seebeck as change in entropy of mixing divided by the charge of the carrier, polarons have twice larger Seebeck contribution $\alpha^{\mathrm{el}}$ mix as bipolaron at the same oxidation level. The energetic parameters in a solid that govern the ratio between the concentration of polaron and bipolaron needs to be investigated in order to find the morphology/chemical structure that provide a high electrical conductivity but keep polarons as main carriers. Note however that polyaniline even in the "insulating" regime, an archetype polaronic system, has a rather poor power factor. This suggests that other contributions to the thermopower are important. The localization of the charge carrier in the "insulating-critical" regime may play a significant role in the electron-phonon coupling. Hence, the vibrational softening contribution $\alpha^{\text {el-ph }}$ vib (Eq. 2.4.4) on a chain or stack of chain might be crucial. It is not yet accurately estimated, neither modeled. The structure change of the polymer chain upon carrying a bipolaron is more important than for a polaron. As a consequence, $\alpha^{\mathrm{el}-\mathrm{ph}}$ vib might be larger for bipolarons than polarons. Hence, the design strategies to optimize ZT must include simultaneously the different contributions to the Seebeck coefficient (Eq.2.4.3).

While the power factor can be readily tuned, the thermal conductivity is always of the same order of magnitude. This parameter if lowered further could also enhance ZT of conducting polymers with high power factors $\left(>300 \mu \mathrm{WK}^{-2} \mathrm{~m}^{-1}\right)$. Both the charge transport and the heat transport are favored in crystalline domains. The ideal crystalline structure is the one that facilitates the electronic transport and simultaneously hinders the heat transport in the same direction. The balance between the charge transport along or perpendicular to the polymer chains in a stack depends on the their dimensions (molecular weight $\mathrm{Mw}$ ). Playing with $\mathrm{Mw}$ and self-organization might offer avenues for $\sigma / \kappa$ ratio optimization. The distribution of the phonon-mean free paths in amorphous and semicrystalline polymers is not known, but the average mean free path is expected to be much smaller than $10 \mathrm{~nm}$ at room temperature. Hence, it's no use to follow the same strategy as tha for inorganic thermoelectrics, i.e. to decrease the mean free path via scattering effects in nanostructures. However, nanostructures might be a strategy to control the the 
electrical transport ${ }^{132}$ regime. A way to optimize $\sigma / \kappa$ might be explored by studying the impact of the counterions (nature and concentration) on the thermal conductivity. Doping of a conducting polymer is accompanied by the incorporation of counterions into the polymer bulk, which expands its volume and affects its density $\left(\rho=\sim 0.5-2 \mathrm{gcm}^{-3}\right)$. This effect is translated in a mechanical motion in polymer microactuators ${ }^{133}$. Doping introduces many long-range electrostatic interactions that modify the cohesion energy and the interaction potential between adjacent polymer chains, as well as the collective vibrational motions. Hence, doping is expected to alter the specific heat and the nature of the counterions might affect the thermal conductivity.

In conducting polymer-insulator-polymer blends ${ }^{89}$ or in CNT-polymer composite ${ }^{134}$ with percolation paths made of only the conducting materials, the Seebeck coefficient is the same as that of the bulk conducting material. The Seebeck coefficient is "weighted" by the conductivity of the conducting paths. Hence, the realization of efficient thermoelectric polymer-based composites requires the formation of conducting paths composed of both inorganic and organic constituents. One strategy to create thermoelectrically efficient polymer-inorganic nanocomposites is to use inorganic nanowires with low thermal conductivity and high electrical conductivity, and decorate them with an organic conducting shell (e.g. via self-assembled monolayer methods). This appears to be a safer route than fabricating a composite with high load inorganic fillers without creating percolation paths. Another requirement concerns with the energy barrier that should be as small as possible for the charge carriers travelling through organic and inorganic phases. In order words, the Fermi levels of the organic and inorganic parts have to match. Tuning of the Fermi level of the inorganic semiconductor and the conducting polymer matrix can be carried out independently. However, the control of the doping level in inorganic nanostrcutures is still an emerging research area ${ }^{135}$. Note that a major obstacle for achieving a thermoelectric polymer composite is the presence of an oxide insulating layer at the surface of most semiconducting inorganic nanostructures. Hence the strategies to remove the oxide and keep the particle oxide free are required.

\section{Conclusion}

The optimization of ZT in conducting polymers is only in its infancy and requires a systematic understanding of the influence of morphology, chemical and electronic structure on three principal thermoelectric parameters. The problem is nontrivial since the thermoelectric properties are related to each other and conducting polymers are known for their morphological complexity and anisotropy of their physical properties. The knowledge available on the structure-charge transport relationship acquired during the last decades on conducting and semiconducting polymers for organic electronics constitutes a strong basis for the development of polymer-based thermoelectrics. A major challenge of this emerging research area is the understanding of the various origins of the Seebeck effect in conducting polymers in order to attain high power factors. Additionally more systematic methods for material thermoelectric properties characterization are required. In order to improve polymer-based organic thermoelectric generators, new manufacturing 
techniques need to be developed to fabricate micro TEGs with many hundreds of 10 to $1000 \mu \mathrm{m}$-long legs. Today, the perspective of large-scale thermoelectric energy harvesting from waste and solar heat may seem obscure, but some important efforts are being made to bring closer the day when it will no longer be just a possibility.

\section{ACKNOWLEDGMENTS}

The authors acknowledge the European Research Council (ERC-starting-grant 307596), the Swedish foundation for strategic research (project: "Nano-material and Scalable TE materials"), the Knut and Alice Wallenberg foundation (project "Power paper"), The Swedish Energy Agency and the Advanced Functional Materials Center at Linköping University.

\section{References :}

1. N. Savage, Nat Photon, 2009, 3, 541-542.

2. H. Bottner, J. Nurnus, A. Gavrikov, G. Kuhner, M. Jagle, C. Kunzel, D. Eberhard, G. Plescher, A. Schubert and K. H. Schlereth, Microelectromechanical Systems, Journal of, 2004, 13, 414-420.

3. W. A. Wong, Anderson, David J., Tuttle, Karen L., Tew, Roy C, NASA Radioisotope Power Conversion Technology NRA Overview, 2005.

4. H. N. M. Kishi, T. Hamao, M. Yamamoto, S. Sudou, M. Mandai, S., Micro thermoelectric modules and their application to wristwatches as an energy source, 1999.

5. J. P. Heremans, C. M. Thrush, D. T. Morelli and M.-C. Wu, Physical Review Letters, 2002, 88, 216801.

6. M. Assael, S. Botsios, K. Gialou and I. Metaxa, International Journal of Thermophysics, 2005, 26, 1595-1605.

7. G. J. Snyder and E. S. Toberer, Nat Mater, 2008, 7, 105-114.

8. W. M. Yim and F. D. Rosi, Solid-State Electronics, 1972, 15, 1121-1140.

9. L. A. K. V. L. Kuznetsov, A. E. Kaliazin, and D. M. Rowe, Journal of Applied Physics 2000, 87, 5.

10. G. J. Poon, in Semiconductors and Semimetals, ed. M. T. Terry, Elsevier, 2001, vol. Volume 70, pp. 37-75.

11. B. A. Fleurial J-P, Caillat T, Morelli DT, Meisner GP, High figure of merit in Ce filled skutterudites Pasadena, CA, USA 1996.

12. W. Luo, H. Li, Y. Yan, Z. Lin, X. Tang, Q. Zhang and C. Uher, Intermetallics, 2011, 19, 404-408.

13. M. Shikano and R. Funahashi, Applied Physics Letters, 2003, 82, 1851-1853.

14. Y. L. a. R. F. Jian He, Journal of Materials Research, 2011, 26, 10.

15. S. P. Armes, M. Aldissi, M. Hawley, J. G. Beery and S. Gottesfeld, Langmuir, 1991, 7, 1447-1452.

16. J. Ashkenazi, W. E. Pickett, H. Krakauer, C. S. Wang, B. M. Klein and S. R. Chubb, Physical Review Letters, 1989, 62, 2016-2019.

17. Y.-J. Cheng, S.-H. Yang and C.-S. Hsu, Chemical Reviews, 2009, 109, 5868-5923.

18. H. Sirringhaus, T. Kawase, R. H. Friend, T. Shimoda, M. Inbasekaran, W. Wu and E. P. Woo, Science, 2000, 290, 2123-2126. 
19. C. A. Hunter and J. K. M. Sanders, Journal of the American Chemical Society, 1990, 112, 5525-5534.

20. P. J. Brown, D. S. Thomas, A. Köhler, J. S. Wilson, J.-S. Kim, C. M. Ramsdale, H. Sirringhaus and R. H. Friend, Physical Review B, 2003, 67, 064203.

21. H. Sirringhaus, P. J. Brown, R. H. Friend, M. M. Nielsen, K. Bechgaard, B. M. W. Langeveld-Voss, A. J. H. Spiering, R. A. J. Janssen, E. W. Meijer, P. Herwig and D. M. de Leeuw, Nature, 1999, 401, 685-688.

22. J. Cornil, D. A. dos Santos, X. Crispin, R. Silbey and J. L. Brédas, Journal of the American Chemical Society, 1998, 120, 1289-1299.

23. J. Cornil, D. Beljonne, J. P. Calbert and J. L. Brédas, Advanced Materials, 2001, 13, 1053-1067.

24. X. Crispin, J. Cornil, R. Friedlein, K. K. Okudaira, V. Lemaur, A. Crispin, G. Kestemont, M. Lehmann, M. Fahlman, R. Lazzaroni, Y. Geerts, G. Wendin, N. Ueno, J.-L. Brédas and W. R. Salaneck, Journal of the American Chemical Society, 2004, 126, 11889-11899.

25. G. Koller, S. Berkebile, M. Oehzelt, P. Puschnig, C. Ambrosch-Draxl, F. P. Netzer and M. G. Ramsey, Science, 2007, 317, 351-355.

26. V. Coropceanu, J. Cornil, D. A. da Silva Filho, Y. Olivier, R. Silbey and J.-L. Brédas, Chemical Reviews, 2007, 107, 926-952.

27. W. Zhang, J. Smith, S. E. Watkins, R. Gysel, M. McGehee, A. Salleo, J. Kirkpatrick, S. Ashraf, T. Anthopoulos, M. Heeney and I. McCulloch, Journal of the American Chemical Society, 2010, 132, 11437-11439.

28. H. Yan, Z. Chen, Y. Zheng, C. Newman, J. R. Quinn, F. Dotz, M. Kastler and A. Facchetti, Nature, 2009, 457, 679-686.

29. H. T. Yi, Y. Chen, K. Czelen and V. Podzorov, Advanced Materials, 2011, 23, 5807-5811.

30. A. Lv, S. R. Puniredd, J. Zhang, Z. Li, H. Zhu, W. Jiang, H. Dong, Y. He, L. Jiang, Y. Li, W. Pisula, Q. Meng, W. Hu and Z. Wang, Advanced Materials, 2012, 24, 2626-2630.

31. A. J. Heeger, The Journal of Physical Chemistry $B, 2001, \mathbf{1 0 5}, 8475-8491$.

32. H. Shirakawa, Angewandte Chemie International Edition, 2001, 40, 25742580.

33. A. G. MacDiarmid, Angewandte Chemie International Edition, 2001, 40, 25812590.

34. T. Yamamoto, T. Maruyama, Z.-H. Zhou, T. Ito, T. Fukuda, Y. Yoneda, F. Begum, T. Ikeda and S. Sasaki, Journal of the American Chemical Society, 1994, 116, $4832-4845$.

35. E. C. M. C. Edward S. Chen, Neeta Sane, Laura Talley, Nancy Kozanecki, Shawn Shulze, Journal of Chemical Physics, 1999, 110, 11.

36. Z.-X. L. N. Basescu, D. Moses, A. J. Heeger, H. Naarmann, N. Theophilou, Nature, 1987, 327, 2.

37. Y. Xuan, X. Liu, S. Desbief, P. Leclère, M. Fahlman, R. Lazzaroni, M. Berggren, J. Cornil, D. Emin and X. Crispin, Physical Review B, 2010, 82, 115454.

38. J.-C. Chiang and A. G. MacDiarmid, Synthetic Metals, 1986, 13, 193-205.

39. G. Zotti, S. Zecchin, G. Schiavon, F. Louwet, L. Groenendaal, X. Crispin, W. Osikowicz, W. Salaneck and M. Fahlman, Macromolecules, 2003, 36, 33373344.

40. L. Groenendaal, F. Jonas, D. Freitag, H. Pielartzik and J. R. Reynolds, Advanced Materials, 2000, 12, 481-494. 
41. O. Bubnova, Z. U. Khan, A. Malti, S. Braun, M. Fahlman, M. Berggren and X. Crispin, Nat Mater, 2011, 10, 429-433.

42. K. Lee, S. Cho, S. Heum Park, A. J. Heeger, C.-W. Lee and S.-H. Lee, Nature, 2006, 441, 65-68.

43. X. Crispin, F. L. E. Jakobsson, A. Crispin, P. C. M. Grim, P. Andersson, A. Volodin, C. van Haesendonck, M. Van der Auweraer, W. R. Salaneck and M. Berggren, Chemistry of Materials, 2006, 18, 4354-4360.

44. Y. Cao, J. Qiu and P. Smith, Synthetic Metals, 1995, 69, 187-190.

45. K. Mizoguchi, M. Nechtschein, J. P. Travers and C. Menardo, Physical Review Letters, 1989, 63, 66-69.

46. J. L. Bredas and G. B. Street, Accounts of Chemical Research, 1985, 18, 309-315.

47. D. Emin, Physical Review B, 1996, 53, 1260-1268.

48. S. Stafström, J. L. Brédas, A. J. Epstein, H. S. Woo, D. B. Tanner, W. S. Huang and A. G. MacDiarmid, Physical Review Letters, 1987, 59, 1464-1467.

49. D. Beljonne, J. Cornil, H. Sirringhaus, P. J. Brown, M. Shkunov, R. H. Friend and J. L. Brédas, Advanced Functional Materials, 2001, 11, 229-234.

50. P. M. Beaujuge and J. R. Reynolds, Chemical Reviews, 2010, 110, 268-320.

51. J.-L. Brédas, J. Cornil and A. J. Heeger, Advanced Materials, 1996, 8, 447-452.

52. B. Xu and S. Holdcroft, Macromolecules, 1993, 26, 4457-4460.

53. C. Tanase, P. W. M. Blom and D. M. de Leeuw, Physical Review B, 2004, 70, 193202.

54. V. I. Arkhipov, E. V. Emelianova, P. Heremans and H. Bässler, Physical Review $B, 2005,72,235202$.

55. G. D. H. Shimotani, Y. Iwasa, Applied Physics Letters 2005, 86, 3.

56. Y. Xia, K. Sun and J. Ouyang, Advanced Materials, 2012, 24, 2436-2440.

57. P. W. Anderson, Physical Review, 1958, 109, 1492-1505.

58. P. Sheng and J. Klafter, Physical Review B, 1983, 27, 2583-2586.

59. A. J. Epstein, J. M. Ginder, F. Zuo, H. S. Woo, D. B. Tanner, A. F. Richter, M. Angelopoulos, W. S. Huang and A. G. MacDiarmid, Synthetic Metals, 1987, 21, 63-70.

60. A. B. Kaiser and S. C. Graham, Synthetic Metals, 1990, 36, 367-380.

61. R. L. E. T A Skotheim, J R Reynolds,, Handbook of conducting polymer, Marcel Dekker, New-York 1998.

62. S. Stafström, Physical Review B, 1995, 51, 4137-4142.

63. V. N. Prigodin and K. B. Efetov, Physical Review Letters, 1993, 70, 2932-2935.

64. S. N F Mott, E A Davis, Electronic processes in non-crystalline materials, Oxford : Clarendon Press: Oxford University Press, New-York, 1979.

65. G. Kopidakis, C. M. Soukoulis and E. N. Economou, Europhysics Letters, 1996, 33, 459-464.

66. D. Emin and T. Holstein, Physical Review Letters, 1976, 36, 323-326.

67. A. L. Efros and B. I. Shklovskii, Journal of Physics C: Solid State Physics, 1975, 8, L49-L51.

68. N. F. Mott, Journal of Non-Crystalline Solids, 1968, 1, 1-17.

69. J. Joo, Z. Oblakowski, G. Du, J. P. Pouget, E. J. Oh, J. M. Wiesinger, Y. Min, A. G. MacDiarmid and A. J. Epstein, Physical Review B, 1994, 49, 2977-2980.

70. R. S. Kohlman, J. Joo, Y. G. Min, A. G. MacDiarmid and A. J. Epstein, Physical Review Letters, 1996, 77, 2766-2769.

71. C. Herring, Physical Review, 1954, 96, 1163-1187.

72. A. B. Kaiser, Reports on Progress in Physics, 2001, 64, 1-49.

73. D. Emin, Physical Review B, 1999, 59, 6205-6210. 
74. R. W. U. R. R. Heikes, Thermoelectricity: Science and Engineering, Interscience, London, 1961.

75. T. L. Aselage, D. Emin and S. S. McCready, Physical Review B - Condensed Matter and Materials Physics, 2001, 64, 0543021-0543028.

76. A. von Mühlenen, N. Errien, M. Schaer, M. N. Bussac and L. Zuppiroli, Physical Review B, 2007, 75, 115338.

77. Y. Furukawa, Journal of Physical Chemistry, 1996, 100, 15644-15653.

78. D. K. C. MacDonald, Thermoelectricity: An introduction to the principle, Dover, 2006.

79. C. O. Yoon, M. Reghu, D. Moses and A. J. Heeger, Physical Review B, 1994, 49, 10851-10863.

80. C. O. Yoon, M. Reghu, D. Moses, A. J. Heeger, Y. Cao, T. A. Chen, X. Wu and R. D. Rieke, Synthetic Metals, 1995, 75, 229-239.

81. Y. W. Park, W. K. Han, C. H. Choi and H. Shirakawa, Physical Review B, 1984, 30, 5847-5851.

82. T. Hasan Gilani, T. Masui, G. Yu. Logvenov and T. Ishiguro, Synthetic Metals, 1996, 78, 327-331.

83. Y. W. Park, Y. S. Lee, C. Park, L. W. Shacklette and R. H. Baughman, Solid State Communications, 1987, 63, 1063-1066.

84. A. B. Kaiser, Physical Review B, 1987, 35, 4677-4681.

85. T. H. Gilani and T. Ishiguro, Synthetic Metals, 1997, 84, 845-846.

86. A. B. Kaiser, Advanced Materials, 2001, 13, 927-941.

87. A. B. Kaiser, Physical Review B, 1989, 40, 2806-2813.

88. W. Pukacki, J. Płocharski and S. Roth, Synthetic Metals, 1994, 62, 253-256.

89. C. O. Yoon, M. Reghu, D. Moses, A. J. Heeger and Y. Cao, Synthetic Metals, 1994, 63, 47-52.

90. M. Rusu, A. Stanciu, V. Bulacovschi, G. G. Rusu, B. Mihaela and G. I. Rusu, Thin Solid Films, 1998, 326, 256-262.

91. H. I. S. Ukai, S. Kuroda, presented in part at the the International Conference for the Science and Technology of Synthetic Metals (ICSM

2004), New South Wales, Australia, 2004.

92. O. Parfenov and F. Shklyaruk, Semiconductors, 2007, 41, 1021-1026.

93. T. E. Whall, Journal of Physics C: Solid State Physics, 1981, 14, L887-L893.

94. P. M. Chaikin and G. Beni, Physical Review B, 1976, 13, 647-651.

95. S. D. Baranovskii, I. P. Zvyagin, H. Cordes, S. Yamasaki and P. Thomas, physica status solidi (b), 2002, 230, 281-288.

96. N. S. H. Yan, N. Toshima, Journal of Thermal Analysis and Calorimetry, 2002, 69, 6.

97. D. Moses and A. Denenstein, Physical Review B, 1984, 30, 2090-2097.

98. P. B. Kaul, K. A. Day and A. R. Abramson, Journal of Applied Physics, 2007, 101, 083507-083507.

99. K. K. Lee, A. S. Alexandrov and W. Y. Liang, Physical Review Letters, 2003, 90, 217001.

100. D. G. Cahill and R. O. Pohl, Physical Review B, 1987, 35, 4067-4073.

101. J. Michalski, Physical Review B, 1992, 45, 7054-7065.

102. S. Lepri, R. Livi and A. Politi, Physics Reports, 2003, 377, 1-80.

103. S. Shenogin, A. Bodapati, P. Keblinski and A. J. H. McGaughey, Journal of Applied Physics, 2009, 105, 034906-034906.

104. in Prediction of Polymer Properties, CRC Press, 2002. 
105. S. Belin, K. Behnia, M. Ribault, A. Deluzet and P. Batail, Synthetic Metals, 1999, 103, 2046-2047.

106. K. Torizuka, H. Tajima, Y. Kawamura, H. Sawa and T. Yamamoto, Journal of Physics and Chemistry of Solids, 2005, 66, 1575-1578.

107. Y. S. Ju, K. Kurabayashi and K. E. Goodson, Thin Solid Films, 1999, 339, 160 164.

108. A. G. Gibson, D. Greig, M. Sahota, I. M. Ward and C. L. Choy, Journal of Polymer Science: Polymer Letters Edition, 1977, 15, 183-192.

109. B. Poudel, Q. Hao, Y. Ma, Y. Lan, A. Minnich, B. Yu, X. Yan, D. Wang, A. Muto, D. Vashaee, X. Chen, J. Liu, M. S. Dresselhaus, G. Chen and Z. Ren, Science, 2008, 320, 634-638.

110. Y. Nogami, H. Kaneko, T. Ishiguro, A. Takahashi, J. Tsukamoto and N. Hosoito, Solid State Communications, 1990, 76, 583-586.

111. J. P. W. Pukacki, S. Rothc Anisotropy of Charge Transport Properties in Highly Conductive Oriented Polyacetylene, Capri, Italy, 1993.

112. N. Mateeva, H. Niculescu, J. Schlenoff and L. R. Testardi, Journal of Applied Physics, 1998, 83, 3111-3117.

113. N. Toshima, Macromolecular Symposia, 2002, 186, 81-86.

114. R. d. B. Aïch, N. Blouin, A. l. Bouchard and M. Leclerc, Chemistry of Materials, 2009, 21, 751-757.

115. Y. Hiroshige, M. Ookawa and N. Toshima, Synthetic Metals, 2006, 156, 13411347.

116. Y. Hiroshige, M. Ookawa and N. Toshima, Synthetic Metals, 2007, 157, 467474.

117. Y. Sun, P. Sheng, C. Di, F. Jiao, W. Xu, D. Qiu and D. Zhu, Advanced Materials, 2012, 24, 932-937.

118. J. Ouyang, Q. Xu, C.-W. Chu, Y. Yang, G. Li and J. Shinar, Polymer, 2004, 45, 8443-8450.

119. F.-X. Jiang, J.-K. Xu, B.-Y. Lu, Y. Xie, R.-J. Huang and L.-F. Li, Chinese Physics Letters, 2008, 25, 2202.

120. M. B. O. Bubnova, Y. Geerts, X. Crispin, unpublished.

121. T.-C. Tsai, H.-C. Chang, C.-H. Chen and W.-T. Whang, Organic Electronics, 2011, 12, 2159-2164.

122. M. S. Dresselhaus, G. Chen, M. Y. Tang, R. G. Yang, H. Lee, D. Z. Wang, Z. F. Ren, J. P. Fleurial and P. Gogna, Advanced Materials, 2007, 19, 1043-1053.

123. K. C. See, J. P. Feser, C. E. Chen, A. Majumdar, J. J. Urban and R. A. Segalman, Nano Letters, 2010, 10, 4664-4667.

124. B. Zhang, J. Sun, H. E. Katz, F. Fang and R. L. Opila, ACS Applied Materials \& Interfaces, 2010, 2, 3170-3178.

125. D. Kim, Y. Kim, K. Choi, J. C. Grunlan and C. Yu, ACS Nano, 2009, 4, 513-523.

126. F. H. Gojny, M. H. G. Wichmann, B. Fiedler, I. A. Kinloch, W. Bauhofer, A. H. Windle and K. Schulte, Polymer, 2006, 47, 2036-2045.

127. Y. Ryu, D. Freeman and C. Yu, Carbon, 2011, 49, 4745-4751.

128. Y. Xuan, M. Sandberg, M. Berggren and X. Crispin, Organic Electronics, 2012, 13, 632-637.

129. J. Wüsten and K. Potje-Kamloth, Journal of Physics D: Applied Physics, 2008, 41.

130. L. Zuppiroli, M. N. Bussac, S. Paschen, O. Chauvet and L. Forro, Physical Review $B, 1994, \mathbf{5 0}, 5196-5203$.

131. J. O. Sofo and G. D. Mahan, Physical Review B, 1994, 49, 4565-4570. 
132. J. L. Duvail, Y. Long, S. Cuenot, Z. Chen and C. Gu, Applied Physics Letters, 2007, 90.

133. E. W. H. Jager, E. Smela and O. Inganas, Science, 2000, 290, 1540-1545.

134. C. Yu, Y. S. Kim, D. Kim and J. C. Grunlan, Nano Letters, 2008, 8, 4428-4432.

135. S. C. Erwin, L. Zu, M. I. Haftel, A. L. Efros, T. A. Kennedy and D. J. Norris, Nature, 2005, 436, 91-94. 